Article

\title{
The Influence of Hydrothermal Fluids on the REY-Rich Deep-Sea Sediments in the Yupanqui Basin, Eastern South Pacific Ocean: Constraints from Bulk Sediment Geochemistry and Mineralogical Characteristics
}

\author{
Tiancheng Zhou ${ }^{1,2}$, Xuefa Shi ${ }^{1,2}, *$, Mu Huang ${ }^{1,2} \oplus$, Miao Yu ${ }^{1,2}$, Dongjie Bi ${ }^{1,2}$, \\ Xiangwen Ren ${ }^{1,2}$, Gang Yang ${ }^{1,2}$ and Aimei Zhu ${ }^{1,2}$ \\ 1 Key Laboratory of Marine Geology and Metallogeny, First Institute of Oceanography, \\ Ministry of Natural Resources, Qingdao 266061, China; ztc1989@163.com (T.Z.); \\ huangmu@fio.org.cn (M.H.); myu@fio.org.cn (M.Y.); dongjiebi@fio.org.cn (D.B.); \\ renxiangwen@fio.org.cn (X.R.); yanggang@fio.org.cn (G.Y.); zhuaimei@fio.org.cn (A.Z.) \\ 2 Laboratory for Marine Geology, Qingdao National Laboratory for Marine Science and Technology, \\ Qingdao 266061, China \\ * Correspondence: xfshi@fio.org.cn; Tel.: +86-0532-88967491
}

Received: 15 October 2020; Accepted: 15 December 2020; Published: 19 December 2020

\begin{abstract}
Rare earth elements (REEs) and yttrium (Y), together known as REY, are extremely enriched in deep-sea pelagic sediments, attracting much attention as a promising new REY resource. To understand the influence of hydrothermal processes on the enrichment of REY in deep-sea sediments from the eastern South Pacific Ocean, we conducted detailed lithological, bulk sediment geochemical, and in situ mineral geochemical analyses on gravity core sample S021GC17 from the Yupanqui Basin of eastern South Pacific. The REY-rich muds of S021GC17 are dark-brown to black zeolitic clays with REY contents ( $\Sigma R E Y$ ) ranging from 1057 to 1882 ppm (average $1329 \mathrm{ppm}$ ). The REY-rich muds display heavy rare earth elements (HREE) enriched patterns, with obvious depletions in Ce, and positive anomalies of Eu in Post-Archean Australian Shale (PAAS)-normalized REE diagrams. In contrast, the muds of S021GC17 show light rare earth elements (LREE) enriched patterns and positive anomalies of $\mathrm{Ce}$ and $\mathrm{Eu}$ in the seawater-normalized REE diagrams. Total REY abundances in the core show positive correlations with $\mathrm{CaO}, \mathrm{P}_{2} \mathrm{O}_{5}, \mathrm{Fe}_{2} \mathrm{O}_{3}$, and $\mathrm{MnO}$ concentrations. In situ analyses of trace element contents by laser ablation-inductively coupled plasma-mass spectrometry (LA-ICP-MS) demonstrate that bioapatite fossils contain high REY concentrations (998 to 22,497 ppm, average $9123 \mathrm{ppm}$ ), indicating that they are the primary carriers of REY. The in situ $\mathrm{Nd}$ isotope values of bioapatites are higher than the average values of seawater in Pacific Ocean. Fe-Mn micronodules are divided into hydrogenetic and diagenetic types, which have average REY concentrations of 1586 and $567 \mathrm{ppm}$, respectively. The high contents of Fe-Mn-Ba-Co-Mo, the positive correlations between $\Sigma$ REY and Fe-Mn, the ratios of $\mathrm{Fe} / \mathrm{Ti}$ and $\mathrm{Al} /(\mathrm{Al}+\mathrm{Fe}+\mathrm{Mn})$, and the LREE-enriched patterns in the REY-rich muds, combined with high Nd isotope values shown by bioapatite fossils, strongly indicate that the hydrothermal fluids have played an important role in the formation of the REY-rich sediments in the eastern South Pacific Ocean.
\end{abstract}

Keywords: deep-sea sediment; eastern South Pacific; bioapatite; hydrothermal fluids; LA-(MC)-ICP-MS 


\section{Introduction}

Rare earth elements and yttrium (REY) are critical materials in many present-day industrial products, including applications in the new energy, electronics, and medical fields. Recently, deep-sea sediments with high concentrations of REY, called "REY-rich mud", have attracted attention as a promising new resource of REY [1-3].

REY-rich mud is defined as a deep-sea sediment that contains more than $700 \mathrm{ppm}$ of total REY [4], which is higher than the grade of ion-adsorption-type ore deposits in southern China $[5,6]$. REY-rich muds from the eastern South Pacific and the central North Pacific have been firstly reported as potential resources with significant amounts of REY [1]. Furthermore, thick layers of REY-rich muds with comparable $\Sigma$ REY contents have been found in the Indian Ocean, and they share similar geochemical and mineralogical features with those in the Pacific Ocean [7,8]. In 2016, extremely high REY-rich muds containing $>7000 \mathrm{ppm}$ of $\Sigma$ REY were reported within the Japanese exclusive economic zone (EEZ) around Minamitorishima Island in the western North Pacific Ocean [9,10]. Most recently, our group discovered large areas of REY-rich muds in the eastern South Pacific Ocean in 2018 [4].

The influence of hydrothermal fluids on the chemistry of REY-rich muds is debated. In the eastern South Pacific Ocean, the formation of REY-rich muds appears to be strongly associated with hydrothermal activity along the East Pacific Rise (EPR). Kato et al. [1] proposed that hydrothermal iron oxyhydroxides that precipitate from hydrothermal plumes are the main carrier of REY in the Pacific. Moreover, the far-field spreading of hydrothermal plumes from the EPR is consistent with the spatial distribution of REY-rich muds in the Pacific Ocean [11-13]. However, most of the other REY-rich muds reported in the central North Pacific, the western Pacific, and the Indian Ocean, appear to be unrelated to hydrothermal fluids [7-9]. Recent studies using both SEM and laser ablation-inductively coupled plasma-mass spectrometry (LA-ICP-MS) have shown that apatite is certainly the host mineral of REY in the REY-rich muds of the Indian and western North Pacific Oceans, where the hydrothermal influence is minor [7-9,14-16]. Moreover, even in strongly hydrothermal-affected sediments around the EPR, apatite is also the major host of La, according to a preliminary $\mathrm{X}$-ray adsorption fine structure (XAFS) analysis [17]. Thus, the influence of hydrothermal fluids on the REY enrichment mechanism of the deep-sea sediments remains controversial.

The eastern South Pacific Ocean contains large volumes of REY-rich muds, which have $\sum$ REY contents of 1000-2230 ppm with total heavy rare earth elements concentrations ( $\sum$ HREE) of 200-430 ppm [1]. Although bulk sediment analyses of REY-rich muds have been conducted on several samples from this area [1], the host minerals of the REY and their relative abundances remain poorly known. Moreover, no systematic study about the influence of hydrothermal fluids on the REY-rich muds has been conducted.

In this paper we present the results of bulk sediment elemental analyses and data of in situ electron microprobe analysis (EMPA) and laser ablation inductively coupled plasma mass spectrometry (LA-ICP-MS) elemental analyses of bioapatite fossils, Fe-Mn micronodules, and phillipsite included in the REY-rich muds. We also used in situ laser ablation multiple collector inductively coupled plasma mass spectrometry (LA-MC-ICP-MS) Sr-Nd isotope analyses of the bioapatite fossils and multiple collector thermal ionization mass spectrometer (MC-TIMS) Sr-Nd isotope analyses of the muds to characterize the isotopic features of these sediments. These data were used to identify the main mineral carriers of REY and discuss the influence of hydrothermal fluids in the REY enrichment process.

\section{Regional Geology}

The eastern South Pacific Ocean features a large-scale aseismic ridge called the East Pacific Rise (EPR), which generally rises $2000-4000 \mathrm{~m}$ above the ocean floor. The EPR is a typical fast to superfast-spreading ridge with a full spreading rate of $80-200 \mathrm{~mm} /$ year $[18,19]$. The EPR trends roughly N-S through the eastern South Pacific near $110^{\circ} \mathrm{W}$, and it features numerous transform faults on both sides [20]. Hydrothermal and volcanic activity are intense along the EPR, generating small- and large-scale hydrothermal plumes rich in $\delta^{3} \mathrm{He}$, in addition to $\mathrm{Mn}, \mathrm{Fe}$, and methane (Figure 1) [21]. 
Resing et al. [22] proposed that dissolved Fe and Mn from the EPR can be transported thousands of kilometers from the venting site in the eastern South Pacific Ocean. Fitzsimmons et al. [23] calculated that the Fe content in the seawater $2000 \mathrm{~km}$ from the EPR is $1-1.5 \mathrm{nmol} / \mathrm{kg}$, which is significantly higher than that in the Pacific Ocean $(0.4-0.9 \mathrm{nmol} / \mathrm{kg})$.
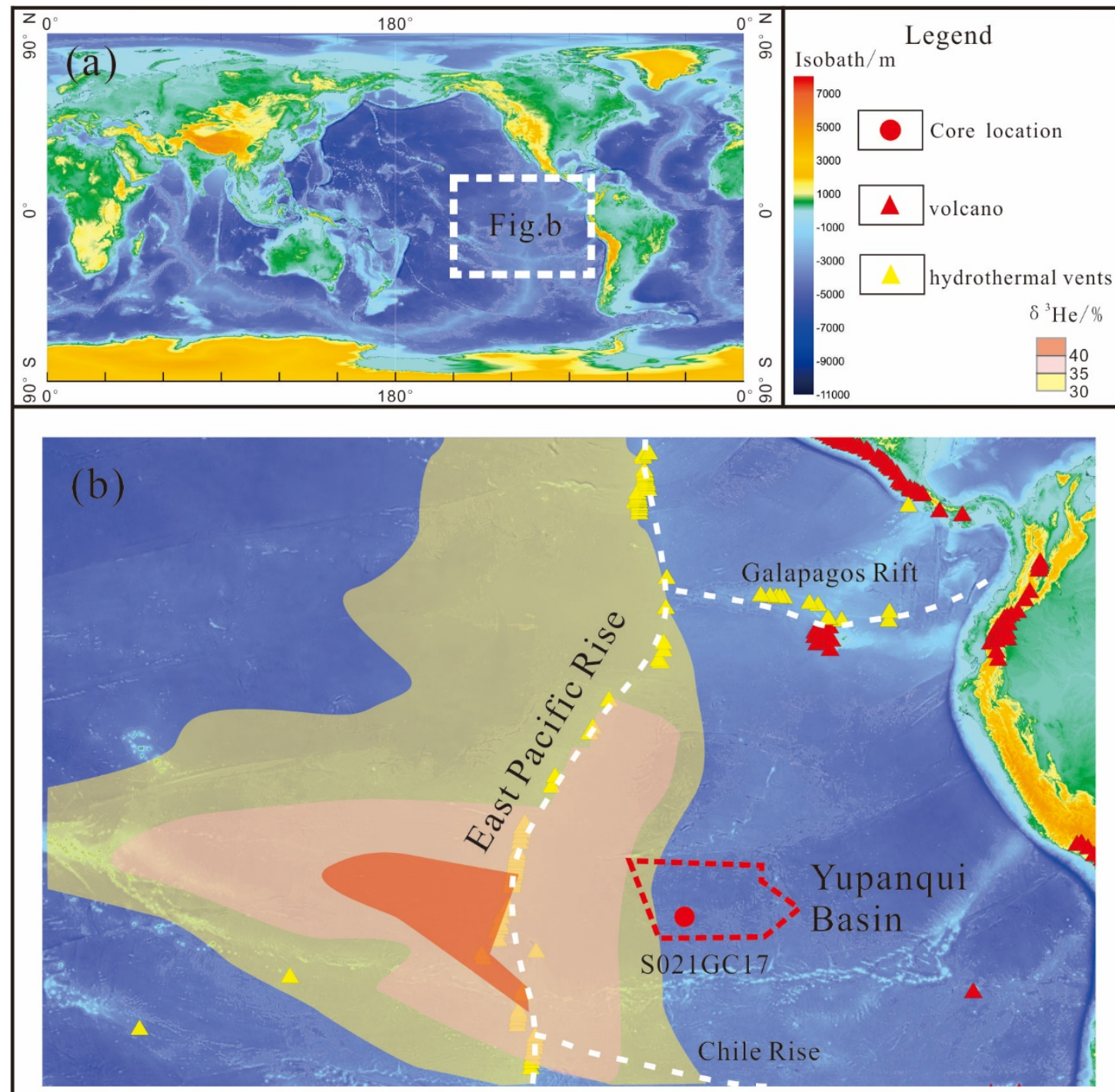

Figure 1. (a). The area of eastern South Pacific Ocean in the map of the world. (b). Location of sampling site S021GC17 in the Yupanqui Basin, eastern South Pacific Ocean (base image from Google Earth).

The eastern South Pacific Ocean has a relatively complex system of seamounts and ridges, and these divide the area into multiple deep-sea basins [24]. To the east of EPR, the Roggeveen and Chile basins are bounded to the north by the Salay Gomez Ridge (SGR) near $25^{\circ} \mathrm{S}$, which is part of the Nazca Plate that extends towards the coast of Chile [20]. The Nazca Plate is surrounded by the East Pacific Rise to the west, Galapagos rift to the north, and Chile-Peru ridges to the south [25], and the Yupanqui Basin lies in the western part of the Nazca Plate (Figure 1b). The age of the basement in the Yupanqui Basin is $<20 \mathrm{Ma}[26]$.

The eastern South Pacific Ocean has rich water masses, which bring in dissolved oxygen and cause sediment decomposition [27]. The North Pacific water masses flow generally towards the Southern Ocean and meet the Antarctic Circumpolar Current (ACC), upstream of Drake Passage. Subantarctic Surface Water (SASW) is found to the north of the Subantarctic Front, and is carried 
northward in the eastern South Pacific Ocean along the rim of the Subtropical Gyre [27]. Low salinity Antarctic Intermediate Water (AAIW) occurs at depths between 400 and $1000 \mathrm{~m}[26,28]$.

\section{Samples and Analytical Methods}

\subsection{Lithological Characteristics}

The gravity piston core S021GC17 was collected from the Yupanqui Basin in the eastern South Pacific Ocean. The water depth was about $4212 \mathrm{~m}$ and the gravity core penetrated $3.04 \mathrm{~m}$ below the seafloor. Samples of core ( $2 \mathrm{~cm}$ thick) were taken at intervals of $10 \mathrm{~cm}$ from top to bottom, and the samples were numbered sequentially from S021GC17 0-2 to S021GC17 302-304. The REY-rich muds of S021GC17 are homogeneous dark-brown to black zeolitic clays (Figure 2a) characterized by high contents of clay minerals (mainly Kaolinite and illite) (50\%), phillipsite (15\%), Fe-Mn micronodules $(15 \%)$, and biogenic apatite $(5 \%)$, in addition to terrigenous detrital materials (quartz and feldspar) $(10 \%)$ and carbonate (5\%) (Figures $2 b, c$ and 3$)$. The proportions of the different constituents were estimated roughly by microscope observation. The biogenic apatite (fish teeth fossils) and Fe-Mn oxide in the muds showed no peaks on X-ray diffraction analyses (Figure 3), indicating an amorphous or low crystallinity habit. The selected 31 samples were sealed immediately in clean polyethylene bags and placed in $4{ }^{\circ} \mathrm{C}$ storage.
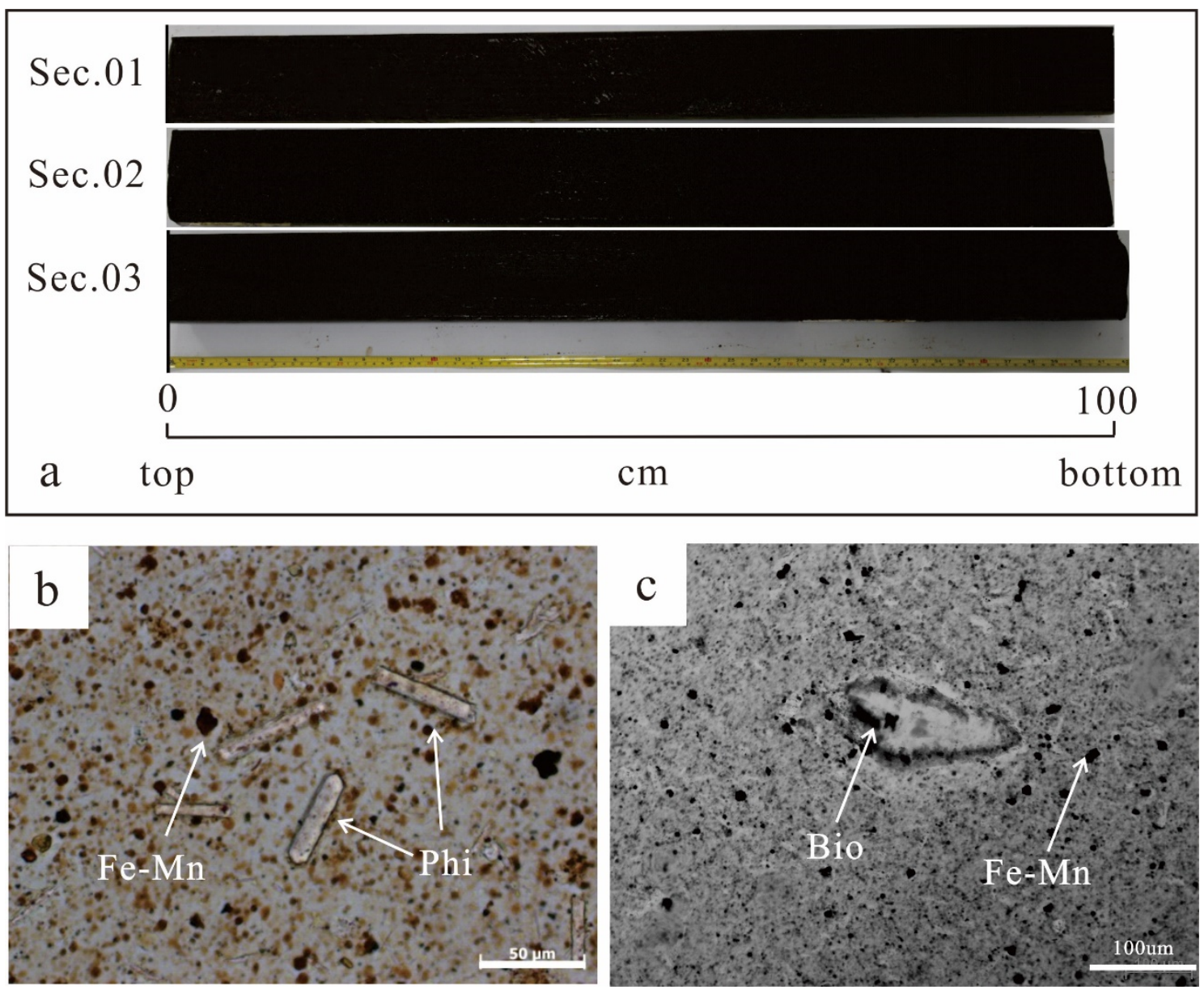

Figure 2. (a) The core material from the S021GC17 site. (b,c) Photomicrographs of sediment samples from the core. Phi = phillipsite; Bio = bioapatite (fish teeth). 

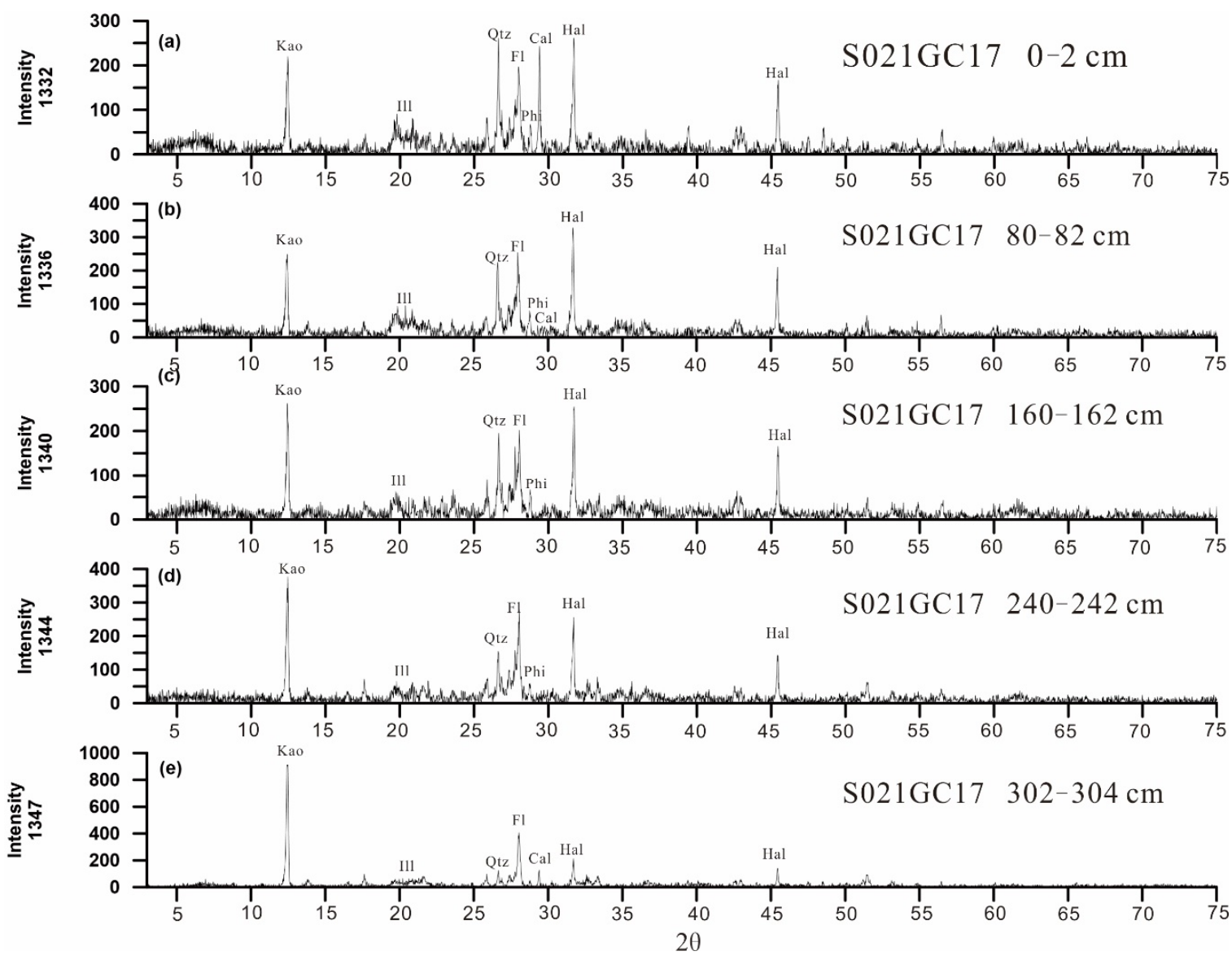

Figure 3. (a-e). X-ray powder diffraction patterns of bulk sediment samples from different depths. Kao = kaolinite; Ill = illite; $\mathrm{Phi}=$ phillipsite; $\mathrm{Qtz}=$ quartz; $\mathrm{Fl}=$ feldspar; $\mathrm{Cal}=$ calcite; $\mathrm{Hal}=$ halite.

\subsection{Bulk Sediment X-ray Diffraction (XRD)}

X-ray diffraction (XRD) analysis can effectively identify the constituents of the sediments. The bulk mineralogy of the samples was assessed using X-ray diffraction (XRD; InXitu Terra, Mountain View, CA, USA) at the Key Laboratory of Marine Geology and Metallogeny, First Institute of Oceanography, Ministry of Natural Resources (MNR), Qingdao, China. Five samples of REY-rich muds were prepared by salt washing, drying, crushing, and filling. The accuracy of the goniometer was better than $0.05^{\circ}(2 \theta)$, instrument resolution better than $60 \%$, and overall stability better than $\pm 1 \%$. Whole patterns from $0^{\circ}$ to $75^{\circ} 2 \theta$ were scanned at $2^{\circ} / \mathrm{min}$ for qualitative analysis of major constituent minerals. Mineral identification was performed using MDI Jade 5.0 software.

\subsection{Bulk Sediment Major-Trace Element Analyses}

Bulk-sediment major and trace element analyses were conducted at the Key Laboratory of Marine Geology and Metallogeny, First Institute of Oceanography, MNR, Qingdao, China. The samples were freeze-dried, powdered to 200 mesh. Each $0.05 \mathrm{~g}$ sample was weighed, placed in a polytetra fluoroethylene digestion tank, dissolved twice in $\mathrm{HF}^{-\mathrm{HNO}_{3}}$ (1:1), and dried again at $190{ }^{\circ} \mathrm{C}$ for $48 \mathrm{~h}$. A total of $3 \mathrm{~mL}$ of $50 \% \mathrm{HNO}_{3}$ was then added to each sample, and the samples were dried at $150{ }^{\circ} \mathrm{C}$ for at least $8 \mathrm{~h}$, removed, and analyzed. Element concentrations were analyzed by inductively coupled plasma-optical emission spectrometry (ICP-OES; for $\mathrm{Al}, \mathrm{Ca}, \mathrm{Fe}, \mathrm{K}, \mathrm{Mg}, \mathrm{Mn}, \mathrm{Na}, \mathrm{P}, \mathrm{Ti}$ ) and inductively coupled plasma-mass spectrometry (ICP-MS; for REY, $\mathrm{Sc}, \mathrm{Cr}, \mathrm{Co}, \mathrm{Ni}$, and $\mathrm{Cu}$ ). Several samples were analyzed in replicate to determine the precision of the measurements, and the elemental composition of the GSD-9 reference standard was measured to confirm the accuracy of the analyses, which was better than $2 \%$ for most elements. 


\subsection{Bulk Sediment Sr-Nd Isotopes}

$\mathrm{Sr}-\mathrm{Nd}$ isotopic signatures can provide important information on the source materials in deep-sea sediments. Bulk sediment $\mathrm{Sr}-\mathrm{Nd}$ isotope analyses were conducted at the same lab in the First Institute of Oceanography, MNR. Rb, Sr, Sm, and Nd concentrations were measured using a Thermo Fisher Scientific Triton Plus multi-collector thermal ionization mass spectrometer (Thermo Fisher Scientific, Waltham, MA, USA). The isotope ratios were corrected for mass fractionation by normalizing to ${ }^{88} \mathrm{Sr} /{ }^{86} \mathrm{Sr}=8.375209$ and ${ }^{146} \mathrm{Nd} /{ }^{144} \mathrm{Nd}=0.7219$. The international standards NBS-987 and JNdi-1 were employed to evaluate instrument stability during data collection. The measured international standard NBS-987 yielded ${ }^{87} \mathrm{Sr} /{ }^{86} \mathrm{Sr}=0.710229 \pm 0.000012(2 \sigma)$ and the JNdi- 1 standard yielded ${ }^{143} \mathrm{Nd} /{ }^{144} \mathrm{Nd}=0.512110 \pm 0.00012(2 \sigma)[29]$.

\subsection{EMPA Major-Element Analyses}

Using the results of the bulk sediment analyses as a guide, typical bioapatite fossils (Figure $4 \mathrm{a}-\mathrm{c}$ ), Fe-Mn micronodules (Figure 4d,g), and phillipsites (Figure 4h,i) were hand-picked using a binocular microscope and cleaned with deionized water. The major-element compositions of minerals were determined by electron microprobe (EMPA-JXA-8230, Jeol Ltd., Tokyo, Japan) at the Key Laboratory of Marine Geology and Metallogeny, First Institute of Oceanography, MNR, China. The microprobe was operated at an accelerating voltage of $15 \mathrm{kV}$ and a beam current of $20 \mathrm{nA}$, with an electron beam defocused to a 1-3 $\mu \mathrm{m}$ spot. Count times were $20 \mathrm{~s}$ for all constituents. Standard specimens used for calibration were $\mathrm{Fe}_{2} \mathrm{O}_{3}$ (for $\mathrm{Fe}$ ), $(\mathrm{Mn}, \mathrm{Ca}) \mathrm{SiO}_{3}$ (for $\mathrm{Mn}$ ), $(\mathrm{Ca}, \mathrm{Na}) \mathrm{Al}(\mathrm{Al}, \mathrm{Si}) \mathrm{Si}_{2} \mathrm{O}_{8}$ (for $\mathrm{Ca}$ and $\mathrm{Al}$ ), $\mathrm{NaAlSi}_{3} \mathrm{O}_{8}$ (for $\mathrm{Na}$, Si and O), $\mathrm{KAlSi}_{3} \mathrm{O}_{8}$ (for K), $\mathrm{PrP}_{5} \mathrm{O}_{14}$ (for P), and $(\mathrm{Mg}, \mathrm{Fe})_{2} \mathrm{SiO}_{4}$ (for $\mathrm{Mg}$ ), and the analyzing crystals used were LIFH (for Fe and Mn), PETH (for K, Ca and P), TAP (for Na, Mg, Al and Si), and LDE1H (for O). While performing the electron microprobe analysis (EMPA), backscattered electron (BSE) images were obtained for representative constituents.

\subsection{LA-ICP-MS REY and Trace Element Analyses}

In situ analysis of REY and trace elements in bioapatite fossils, Fe-Mn micronodules, and phillipsites extracted from the REY-rich muds was conducted at Beijing Createch Testing International (Beijing, China). Laser ablation was performed using an ESI NWR $193 \mathrm{~nm}$ excimer laser ablation system (Omaha, NE, USA). An Analytik Jena Plasma Quant mass spectrometer (Jena, Germany) was used to acquire ion-signal intensities. The spot diameter of the laser beam was $35 \mu \mathrm{m}$ and the ablation frequency was $10 \mathrm{~Hz}$. Helium was used as a carrier gas. Each spot analysis consisted of $\sim 20 \mathrm{~s}$ of background acquisition, $\sim 45 \mathrm{~s}$ of measurement, and $\sim 20 \mathrm{~s}$ of cleaning. Elemental abundances were calibrated against multiple synthetic reference glasses (NIST SRM610, NIST SRM612, BHVO-2G, BCR-2G, and BIR-1G). The suitable internal standards were also carried out for data calibration (Ca was used as an internal standard for bioapatites; Mn for Fe-Mn micronodules; and Al for phillipsites), which corresponded to the $\mathrm{Ca}, \mathrm{Mn}$, and Al contents measured by EMPA of each spot (refer to Supplementary Tables). The following isotope suite was analyzed: ${ }^{139} \mathrm{La},{ }^{140} \mathrm{Ce},{ }^{141} \mathrm{Pr},{ }^{146} \mathrm{Nd},{ }^{147} \mathrm{Sm},{ }^{153} \mathrm{Eu},{ }^{157} \mathrm{Gd},{ }^{159} \mathrm{~Tb},{ }^{163} \mathrm{Dy}$, ${ }^{89} \mathrm{Y},{ }^{165} \mathrm{Ho},{ }^{166} \mathrm{Er},{ }^{169} \mathrm{Tm},{ }^{172} \mathrm{Yb},{ }^{175} \mathrm{Lu},{ }^{137} \mathrm{Ba},{ }^{88} \mathrm{Sr},{ }^{59} \mathrm{Co},{ }^{60} \mathrm{Ni},{ }^{65} \mathrm{Cu}$. The linear correlation between $\mathrm{Ba}$ and $\mathrm{Eu}$ contents is weak, suggesting that the mass interference of $\mathrm{BaO}$ on the $\mathrm{Eu}$ measurement is very minor. 

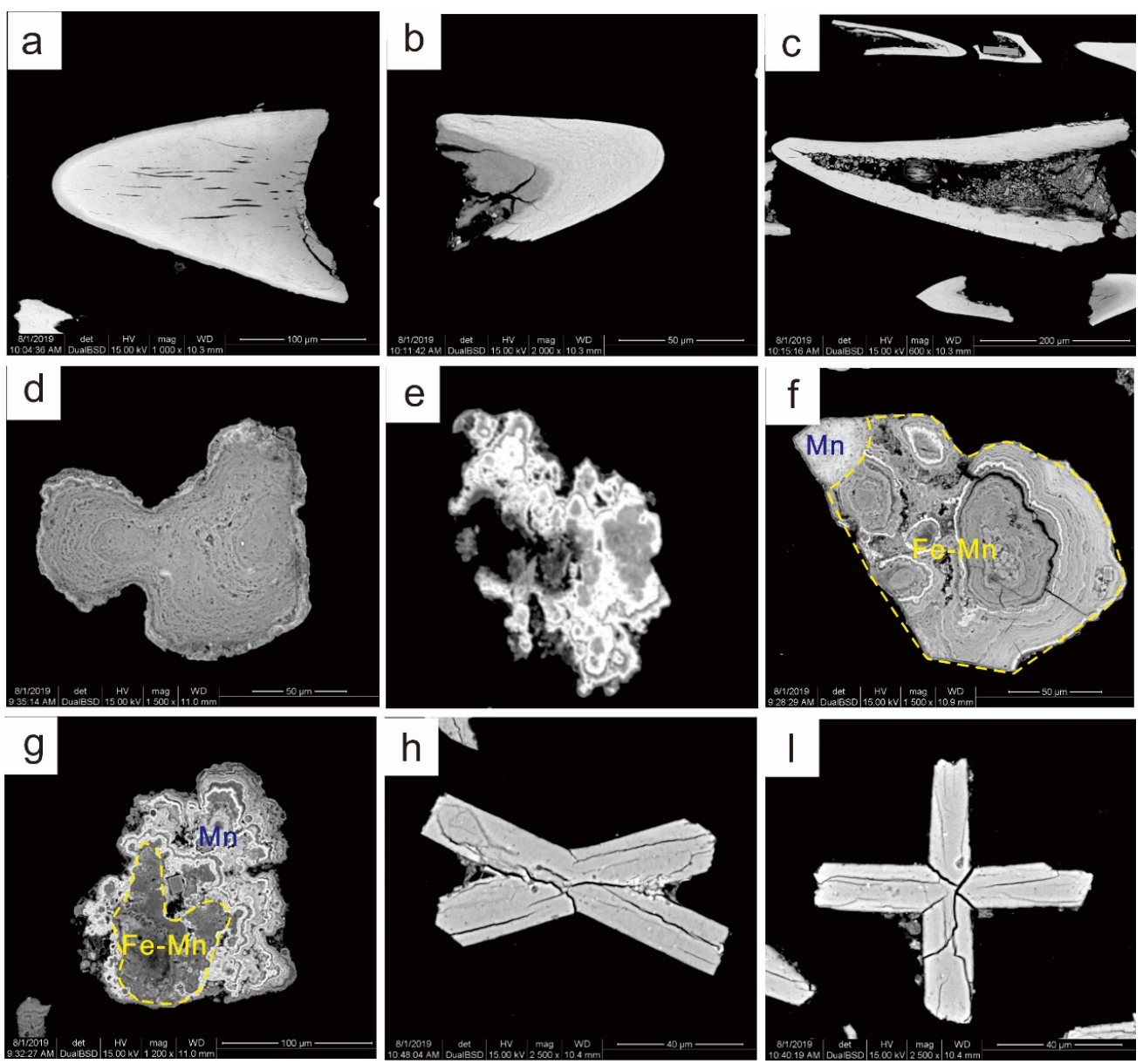

Figure 4. SEM images of the main constituents in the samples. (a-c) Bioapatite (fish teeth). (d) Fe-Mn micronodules. (e) Mn micronodules. (f,g) Mn micronodules in the outer rim of Fe-Mn micronodules. (h,i) Phillipsite.

\subsection{LA-MC-ICP-MS Sr-Nd Isotope Analyses}

In situ Sr-Nd isotope analyses of bioapatite in the REY-rich muds were conducted at Beijing Createch Testing International (Beijing, China). Laser sampling was performed using a Resolution SE $193 \mathrm{~nm}$ excimer laser ablation system. The analytical system involved a Neptune MC-ICPMS. The spot diameter of the laser beam ranged between 35 and $50 \mu \mathrm{m}$ and the ablation frequency was $10 \mathrm{~Hz}$. The aerosol ablated by the laser was transported to the mass spectrometer using helium as a carrier gas. The isotope ratios were corrected for mass fractionation by normalizing to ${ }^{88} \mathrm{Sr} /{ }^{86} \mathrm{Sr}=8.375209$ and ${ }^{147} \mathrm{Sm} /{ }^{149} \mathrm{Sm}=1.0868$. The apatite standard sample Durango was employed to evaluate the MC-ICP-MS instrument stability during data collection, yielding ${ }^{87} \mathrm{Sr} /{ }^{86} \mathrm{Sr}=0.70599 \pm 0.00045(2 \sigma)$ and ${ }^{143} \mathrm{Nd} /{ }^{144} \mathrm{Nd}=0.512478 \pm 0.000029(2 \sigma)[30]$.

\section{Results}

\subsection{Bulk Sediment Major and Trace Element Analyses}

Results of the bulk sediment major and trace element analyses are presented in Table 1a. The REY-rich muds are characterized by high contents of $\mathrm{Fe}_{2} \mathrm{O}_{3}\left(12.70-16.33\right.$ wt.\%) and $\mathrm{Al}_{2} \mathrm{O}_{3}$ (8.53-11.73 wt.\%). Other major elemental oxide contents are $\mathrm{CaO}(2.75-10.45 \mathrm{wt} . \%), \mathrm{MnO}(3.85-5.30$ wt.\%), $\mathrm{Na}_{2} \mathrm{O}$ (5.27-6.46 wt.\%), $\mathrm{K}_{2} \mathrm{O}(2.30-2.61$ wt. $\%), \mathrm{P}_{2} \mathrm{O}_{5}$ (1.30-3.46 wt.\%), and $\mathrm{TiO}_{2}(0.45-0.60$ wt.\%). The Ba contents range from 9187 to 13,410 ppm. The contents of Co and Mo range from 346 to $479 \mathrm{ppm}$ and 49 to $104 \mathrm{ppm}$, respectively. 
Table 1a. Bulk sediment geochemical data for the rare earth elements and yttrium (REY)-rich deep-sea sediments from the Yupanqui Basin.

\begin{tabular}{|c|c|c|c|c|c|c|c|c|c|c|c|c|c|c|c|c|}
\hline Samples & $\begin{array}{c}\text { S021GC17- } \\
0-2\end{array}$ & $\begin{array}{c}\text { S021GC17- } \\
10-12 \\
\end{array}$ & $\begin{array}{c}\text { S021GC17- } \\
20-22\end{array}$ & $\begin{array}{c}\text { S021GC17- } \\
30-32\end{array}$ & $\begin{array}{c}\text { S021GC17- } \\
40-42\end{array}$ & $\begin{array}{c}\text { S021GC17- } \\
50-52\end{array}$ & $\begin{array}{c}\text { S021GC17- } \\
60-62\end{array}$ & $\begin{array}{c}\text { S021GC17- } \\
70-72 \\
\end{array}$ & $\begin{array}{c}\text { S021GC17- } \\
80-82\end{array}$ & $\begin{array}{c}\text { S021GC17- } \\
90-92\end{array}$ & $\begin{array}{c}\text { S021GC17- } \\
100-102\end{array}$ & $\begin{array}{c}\text { S021GC17- } \\
110-112 \\
\end{array}$ & $\begin{array}{c}\text { S021GC17- } \\
120-122\end{array}$ & $\begin{array}{c}\text { S021GC17- } \\
130-132 \\
\end{array}$ & $\begin{array}{c}\text { S021GC17- } \\
140-142\end{array}$ & $\begin{array}{c}\text { S021GC17- } \\
150-152\end{array}$ \\
\hline $\mathrm{Al}_{2} \mathrm{O}_{3}$ & 11.03 & 11.49 & 11.73 & 11.59 & 11.50 & 11.56 & 11.50 & 11.48 & 11.54 & 11.26 & 11.27 & 11.27 & 11.30 & 11.21 & 11.15 & 11.05 \\
\hline $\mathrm{CaO}$ & 5.37 & 3.37 & 2.80 & 2.75 & 2.78 & 2.84 & 2.84 & 2.80 & 2.85 & 2.89 & 3.01 & 2.90 & 2.87 & 2.86 & 2.87 & 2.88 \\
\hline${ }^{\mathrm{T}} \mathrm{Fe}_{2} \mathrm{O}_{3}$ & 12.70 & 13.29 & 13.66 & 13.57 & 13.60 & 13.79 & 13.73 & 13.70 & 13.92 & 13.99 & 14.10 & 14.29 & 14.30 & 14.41 & 14.50 & 14.46 \\
\hline $\mathrm{MnO}$ & 3.85 & 4.04 & 4.23 & 4.21 & 4.19 & 4.22 & 4.21 & 4.22 & 4.31 & 4.40 & 4.47 & 4.56 & 4.60 & 4.67 & 4.70 & 4.67 \\
\hline $\mathrm{K}_{2} \mathrm{O}$ & 2.30 & 2.39 & 2.46 & 2.44 & 2.45 & 2.49 & 2.47 & 2.47 & 2.50 & 2.47 & 2.46 & 2.47 & 2.47 & 2.47 & 2.47 & 2.47 \\
\hline $\mathrm{MgO}$ & 2.99 & 3.08 & 3.18 & 3.12 & 3.16 & 3.18 & 3.16 & 3.13 & 3.19 & 3.15 & 3.14 & 3.16 & 3.15 & 3.17 & 3.18 & 3.18 \\
\hline $\mathrm{Na}_{2} \mathrm{O}$ & 6.04 & 6.04 & 6.37 & 6.20 & 6.35 & 6.38 & 6.38 & 6.25 & 6.46 & 6.32 & 6.13 & 6.16 & 6.12 & 6.08 & 6.08 & 6.13 \\
\hline $\mathrm{P}_{2} \mathrm{O}_{5}$ & 1.30 & 1.33 & 1.37 & 1.40 & 1.43 & 1.46 & 1.46 & 1.43 & 1.44 & 1.46 & 1.51 & 1.51 & 1.52 & 1.53 & 1.56 & 1.61 \\
\hline $\mathrm{TiO}_{2}$ & 0.56 & 0.58 & 0.60 & 0.59 & 0.59 & 0.59 & 0.59 & 0.59 & 0.59 & 0.58 & 0.58 & 0.59 & 0.59 & 0.59 & 0.59 & 0.58 \\
\hline $\mathrm{Ba}$ & 11888 & 12560 & 12537 & 12589 & 12402 & 12270 & 12464 & 12114 & 11732 & 12252 & 11766 & 11903 & 12453 & 12209 & 12466 & 11754 \\
\hline Co & 345.7 & 359.7 & 372.1 & 375.1 & 374.6 & 371.0 & 373.4 & 378.2 & 379.9 & 408.1 & 392.8 & 413.3 & 408.3 & 418.2 & 429.4 & 425.8 \\
\hline Mo & 48.91 & 58.42 & 65.44 & 72.63 & 74.60 & 76.11 & 79.91 & 80.20 & 81.19 & 85.50 & 83.90 & 86.35 & 88.93 & 90.46 & 93.96 & 95.54 \\
\hline $\mathrm{La}$ & 170.51 & 169.98 & 175.01 & 178.66 & 172.71 & 169.55 & 179.99 & 177.03 & 174.40 & 180.98 & 179.91 & 183.35 & 181.84 & 179.07 & 181.63 & 182.72 \\
\hline $\mathrm{Ce}$ & 177.62 & 178.07 & 182.42 & 179.26 & 174.55 & 177.91 & 179.71 & 171.65 & 171.94 & 176.04 & 165.81 & 170.02 & 170.22 & 169.39 & 169.32 & 174.09 \\
\hline $\mathrm{Pr}$ & 37.80 & 39.01 & 39.92 & 40.71 & 40.12 & 39.74 & 41.45 & 39.83 & 40.09 & 42.88 & 42.29 & 43.34 & 42.52 & 42.45 & 42.99 & 42.58 \\
\hline $\mathrm{Nd}$ & 169.07 & 173.56 & 180.48 & 180.28 & 178.66 & 177.16 & 183.94 & 177.41 & 182.73 & 189.69 & 184.93 & 191.85 & 188.83 & 190.23 & 191.04 & 189.31 \\
\hline $\mathrm{Sm}$ & 34.96 & 36.19 & 37.18 & 36.97 & 36.95 & 36.94 & 38.61 & 38.09 & 37.74 & 39.95 & 39.80 & 40.86 & 40.08 & 40.35 & 41.00 & 40.52 \\
\hline $\mathrm{Eu}$ & 14.63 & 15.49 & 15.72 & 15.12 & 15.21 & 15.07 & 15.77 & 15.31 & 14.81 & 16.05 & 15.82 & 16.00 & 15.85 & 15.51 & 15.69 & 15.30 \\
\hline $\mathrm{Gd}$ & 35.00 & 36.17 & 37.98 & 37.76 & 37.80 & 37.28 & 38.59 & 38.24 & 37.81 & 40.85 & 39.92 & 41.64 & 41.13 & 41.16 & 40.68 & 40.09 \\
\hline $\mathrm{Tb}$ & 5.60 & 5.82 & 5.96 & 6.01 & 5.97 & 5.94 & 6.14 & 6.06 & 6.11 & 6.50 & 6.44 & 6.58 & 6.57 & 6.69 & 6.60 & 6.67 \\
\hline Dy & 45.70 & 47.00 & 49.20 & 49.13 & 49.19 & 49.19 & 50.67 & 50.08 & 50.88 & 53.95 & 53.11 & 54.47 & 53.57 & 53.86 & 54.44 & 54.01 \\
\hline Ho & 9.36 & 9.82 & 10.25 & 10.34 & 10.25 & 10.44 & 10.47 & 10.48 & 10.64 & 11.14 & 11.41 & 11.37 & 11.32 & 11.29 & 11.49 & 11.50 \\
\hline Er & 24.12 & 24.93 & 25.91 & 26.02 & 26.07 & 25.90 & 26.60 & 26.20 & 26.39 & 28.08 & 28.09 & 28.28 & 28.19 & 28.64 & 28.91 & 28.63 \\
\hline $\mathrm{Tm}$ & 4.07 & 4.23 & 4.34 & 4.46 & 4.46 & 4.38 & 4.57 & 4.43 & 4.50 & 4.84 & 4.81 & 4.90 & 4.80 & 4.90 & 4.92 & 4.97 \\
\hline $\mathrm{Yb}$ & 24.56 & 25.63 & 26.22 & 26.48 & 26.78 & 26.67 & 27.56 & 26.81 & 27.56 & 29.01 & 28.77 & 29.51 & 28.85 & 28.90 & 29.49 & 29.11 \\
\hline $\mathrm{Lu}$ & 4.07 & 4.25 & 4.46 & 4.47 & 4.53 & 4.56 & 4.55 & 4.61 & 4.60 & 4.96 & 4.92 & 5.02 & 4.77 & 4.91 & 4.87 & 4.83 \\
\hline Y & 299.97 & 306.37 & 324.59 & 331.60 & 331.57 & 328.29 & 333.46 & 339.43 & 344.18 & 366.40 & 355.96 & 368.94 & 352.49 & 355.52 & 367.59 & 370.01 \\
\hline$\sum$ REY & 1057 & 1077 & 1120 & 1127 & 1115 & 1109 & 1142 & 1126 & 1134 & 1191 & 1162 & 1196 & 1171 & 1173 & 1191 & 1194 \\
\hline$\delta \mathrm{Ce}$ & 0.52 & 0.52 & 0.51 & 0.50 & 0.50 & 0.51 & 0.49 & 0.48 & 0.49 & 0.47 & 0.45 & 0.45 & 0.46 & 0.46 & 0.45 & 0.47 \\
\hline$\delta \mathrm{Eu}$ & 1.27 & 1.29 & 1.27 & 1.23 & 1.23 & 1.23 & 1.24 & 1.21 & 1.19 & 1.20 & 1.20 & 1.17 & 1.18 & 1.15 & 1.16 & 1.15 \\
\hline $\mathrm{Y} / \mathrm{Ho}$ & 32.05 & 31.19 & 31.66 & 32.08 & 32.36 & 31.44 & 31.84 & 32.40 & 32.35 & 32.89 & 31.20 & 32.45 & 31.14 & 31.49 & 31.98 & 32.16 \\
\hline $\mathrm{La} / \mathrm{Sm}$ & 4.88 & 4.70 & 4.71 & 4.83 & 4.67 & 4.59 & 4.66 & 4.65 & 4.62 & 4.53 & 4.52 & 4.49 & 4.54 & 4.44 & 4.43 & 4.51 \\
\hline $\mathrm{Sm} / \mathrm{Nd}$ & 0.21 & 0.21 & 0.21 & 0.21 & 0.21 & 0.21 & 0.21 & 0.21 & 0.21 & 0.21 & 0.22 & 0.21 & 0.21 & 0.21 & 0.21 & 0.21 \\
\hline $\mathrm{Sm} / \mathrm{Yb}$ & 1.42 & 1.41 & 1.42 & 1.40 & 1.38 & 1.38 & 1.40 & 1.42 & 1.37 & 1.38 & 1.38 & 1.38 & 1.39 & 1.40 & 1.39 & 1.39 \\
\hline $\mathrm{La} / \mathrm{Yb}$ & 6.94 & 6.63 & 6.67 & 6.75 & 6.45 & 6.36 & 6.53 & 6.60 & 6.33 & 6.24 & 6.25 & 6.21 & 6.30 & 6.20 & 6.16 & 6.28 \\
\hline
\end{tabular}


Table 1b. Bulk sediment geochemical data for the rare earth elements and yttrium (REY)-rich deep-sea sediments from the Yupanqui Basin

\begin{tabular}{|c|c|c|c|c|c|c|c|c|c|c|c|c|c|c|c|}
\hline Samples & $\begin{array}{c}\text { S021GC17- } \\
160-162\end{array}$ & $\begin{array}{c}\text { S021GC17- } \\
170-172\end{array}$ & $\begin{array}{c}\text { S021GC17- } \\
180-182\end{array}$ & $\begin{array}{c}\text { S021GC17- } \\
190-192\end{array}$ & $\begin{array}{c}\text { S021GC17- } \\
200-202\end{array}$ & $\begin{array}{c}\text { S021GC17- } \\
210-212\end{array}$ & $\begin{array}{c}\text { S021GC17- } \\
220-222\end{array}$ & $\begin{array}{c}\text { S021GC17- } \\
230-232\end{array}$ & $\begin{array}{c}\text { S021GC17- } \\
240-242\end{array}$ & $\begin{array}{c}\text { S021GC17- } \\
250-252\end{array}$ & $\begin{array}{c}\text { S021GC17- } \\
260-262\end{array}$ & $\begin{array}{c}\text { S021GC17- } \\
270-272\end{array}$ & $\begin{array}{c}\text { S021GC17- } \\
280-282\end{array}$ & $\begin{array}{c}\text { S021GC17- } \\
290-292\end{array}$ & $\begin{array}{l}\text { S021GC17- } \\
302-304\end{array}$ \\
\hline $\mathrm{Al}_{2} \mathrm{O}_{3}$ & 11.11 & 11.17 & 11.02 & 11.11 & 11.19 & 10.98 & 10.88 & 10.46 & 10.31 & 10.03 & 10.10 & 9.76 & 8.66 & 8.53 & 9.20 \\
\hline $\mathrm{CaO}$ & 2.94 & 3.01 & 3.02 & 3.15 & 3.19 & 3.44 & 3.62 & 3.59 & 3.71 & 3.87 & 4.33 & 4.77 & 8.82 & 10.45 & 6.85 \\
\hline${ }^{\mathrm{T}} \mathrm{Fe}_{2} \mathrm{O}_{3}$ & 14.45 & 14.42 & 14.14 & 14.23 & 14.34 & 14.20 & 14.91 & 14.73 & 14.64 & 14.46 & 14.66 & 15.37 & 14.29 & 15.27 & 16.33 \\
\hline $\mathrm{MnO}$ & 4.73 & 4.70 & 4.69 & 4.76 & 4.79 & 4.75 & 4.95 & 5.00 & 4.97 & 4.88 & 5.02 & 5.30 & 4.98 & 4.83 & 5.11 \\
\hline $\mathrm{K}_{2} \mathrm{O}$ & 2.50 & 2.55 & 2.53 & 2.59 & 2.61 & 2.60 & 2.56 & 2.50 & 2.47 & 2.43 & 2.52 & 2.52 & 2.32 & 2.52 & 2.55 \\
\hline $\mathrm{MgO}$ & 3.22 & 3.24 & 3.19 & 3.21 & 3.23 & 3.20 & 3.27 & 3.22 & 3.14 & 3.04 & 3.00 & 2.99 & 2.65 & 2.48 & 2.48 \\
\hline $\mathrm{Na}_{2} \mathrm{O}$ & 6.28 & 6.34 & 6.09 & 6.06 & 6.08 & 6.11 & 6.08 & 5.90 & 5.90 & 5.88 & 6.02 & 6.00 & 5.43 & 5.27 & 5.43 \\
\hline $\mathrm{P}_{2} \mathrm{O}_{5}$ & 1.63 & 1.68 & 1.76 & 1.88 & 1.90 & 2.10 & 2.24 & 2.27 & 2.36 & 2.45 & 2.71 & 2.96 & 3.17 & 3.28 & 3.46 \\
\hline $\mathrm{TiO}_{2}$ & 0.58 & 0.58 & 0.56 & 0.57 & 0.57 & 0.56 & 0.57 & 0.55 & 0.54 & 0.52 & 0.52 & 0.51 & 0.45 & 0.45 & 0.51 \\
\hline $\mathrm{Ba}$ & 11716 & 11777 & 12667 & 12903 & 13410 & 10556 & 9491 & 9700 & 9187 & 9250 & 9774 & 12284 & 12509 & 12958 & 10607 \\
\hline Co & 427.7 & 419.4 & 418.1 & 433.8 & 418.1 & 437.0 & 464.2 & 479.1 & 450.0 & 447.8 & 465.1 & 457.0 & 428.0 & 392.5 & 413.5 \\
\hline Mo & 94.34 & 88.96 & 92.05 & 97.76 & 96.80 & 93.03 & 96.94 & 104.10 & 96.51 & 90.22 & 83.71 & 81.92 & 82.92 & 85.92 & 92.73 \\
\hline $\mathrm{La}$ & 191.13 & 192.78 & 194.05 & 207.26 & 207.10 & 217.25 & 228.83 & 236.51 & 254.99 & 260.20 & 278.97 & 286.29 & 300.11 & 294.06 & 311.45 \\
\hline $\mathrm{Ce}$ & 166.55 & 163.58 & 162.66 & 164.63 & 164.93 & 155.86 & 159.95 & 158.44 & 156.99 & 148.39 & 155.09 & 140.62 & 134.17 & 136.86 & 151.96 \\
\hline Pr & 44.08 & 43.79 & 44.61 & 45.88 & 46.30 & 47.58 & 50.06 & 49.00 & 51.57 & 49.95 & 53.34 & 51.73 & 52.74 & 53.50 & 57.97 \\
\hline $\mathrm{Nd}$ & 197.25 & 198.93 & 200.21 & 213.74 & 208.73 & 218.59 & 225.26 & 228.34 & 238.30 & 232.70 & 250.10 & 239.60 & 247.63 & 247.42 & 266.70 \\
\hline $\mathrm{Sm}$ & 42.67 & 41.93 & 42.37 & 44.88 & 45.16 & 45.61 & 46.78 & 46.26 & 47.39 & 47.63 & 49.64 & 48.13 & 47.88 & 49.36 & 53.57 \\
\hline $\mathrm{Eu}$ & 16.35 & 15.87 & 16.49 & 17.22 & 17.29 & 16.39 & 15.48 & 15.64 & 15.90 & 15.72 & 16.99 & 17.80 & 18.40 & 19.14 & 18.47 \\
\hline $\mathrm{Gd}$ & 42.22 & 42.28 & 42.31 & 45.55 & 43.86 & 46.34 & 47.17 & 47.14 & 49.35 & 49.55 & 52.27 & 52.59 & 52.74 & 54.20 & 57.26 \\
\hline $\mathrm{Tb}$ & 6.92 & 6.90 & 7.07 & 7.49 & 7.34 & 7.69 & 8.03 & 8.11 & 8.43 & 8.48 & 9.05 & 8.95 & 9.00 & 9.07 & 9.93 \\
\hline Dy & 56.76 & 56.76 & 57.63 & 61.36 & 61.63 & 65.19 & 67.98 & 67.82 & 71.76 & 71.86 & 77.72 & 77.91 & 78.92 & 79.59 & 85.97 \\
\hline Ho & 12.10 & 12.21 & 12.31 & 13.18 & 13.13 & 14.39 & 14.85 & 15.07 & 16.06 & 16.26 & 17.93 & 18.11 & 18.32 & 18.10 & 19.60 \\
\hline Er & 29.99 & 30.04 & 31.29 & 33.59 & 33.59 & 36.05 & 37.77 & 38.14 & 40.95 & 41.44 & 46.01 & 46.50 & 47.23 & 46.75 & 50.65 \\
\hline $\mathrm{Tm}$ & 5.13 & 5.15 & 5.37 & 5.70 & 5.72 & 6.27 & 6.56 & 6.70 & 7.13 & 7.25 & 8.01 & 8.12 & 8.41 & 8.28 & 8.88 \\
\hline $\mathrm{Yb}$ & 30.11 & 30.30 & 31.35 & 33.59 & 33.29 & 36.61 & 38.12 & 39.16 & 41.47 & 42.20 & 47.07 & 47.94 & 48.52 & 48.98 & 52.28 \\
\hline $\mathrm{Lu}$ & 5.14 & 5.12 & 5.24 & 5.62 & 5.57 & 6.12 & 6.31 & 6.49 & 7.11 & 7.17 & 8.02 & 8.13 & 8.42 & 8.45 & 8.98 \\
\hline Y & 387.15 & 391.30 & 407.42 & 444.14 & 430.11 & 494.34 & 530.46 & 537.16 & 561.31 & 585.36 & 684.69 & 701.84 & 735.22 & 703.36 & 728.37 \\
\hline$\sum$ REY & 1234 & 1237 & 1260 & 1344 & 1324 & 1414 & 1484 & 1500 & 1569 & 1584 & 1755 & 1754 & 1808 & 1777 & 1882 \\
\hline$\delta \mathrm{Ce}$ & 0.43 & 0.42 & 0.41 & 0.40 & 0.40 & 0.36 & 0.35 & 0.34 & 0.32 & 0.30 & 0.29 & 0.26 & 0.24 & 0.25 & 0.26 \\
\hline$\delta \mathrm{Eu}$ & 1.16 & 1.14 & 1.18 & 1.15 & 1.17 & 1.08 & 1.00 & 1.01 & 1.00 & 0.98 & 1.01 & 1.08 & 1.11 & 1.13 & 1.01 \\
\hline $\mathrm{Y} / \mathrm{Ho}$ & 32.00 & 32.06 & 33.11 & 33.69 & 32.75 & 34.35 & 35.72 & 35.64 & 34.94 & 35.99 & 38.19 & 38.75 & 40.12 & 38.86 & 37.16 \\
\hline $\mathrm{La} / \mathrm{Sm}$ & 4.48 & 4.60 & 4.58 & 4.62 & 4.59 & 4.76 & 4.89 & 5.11 & 5.38 & 5.46 & 5.62 & 5.95 & 6.27 & 5.96 & 5.81 \\
\hline $\mathrm{Sm} / \mathrm{Nd}$ & 0.22 & 0.21 & 0.21 & 0.21 & 0.22 & 0.21 & 0.21 & 0.20 & 0.20 & 0.20 & 0.20 & 0.20 & 0.19 & 0.20 & 0.20 \\
\hline $\mathrm{Sm} / \mathrm{Yb}$ & 1.42 & 1.38 & 1.35 & 1.34 & 1.36 & 1.25 & 1.23 & 1.18 & 1.14 & 1.13 & 1.05 & 1.00 & 0.99 & 1.01 & 1.02 \\
\hline $\mathrm{La} / \mathrm{Yb}$ & 6.35 & 6.36 & 6.19 & 6.17 & 6.22 & 5.93 & 6.00 & 6.04 & 6.15 & 6.17 & 5.93 & 5.97 & 6.19 & 6.00 & 5.96 \\
\hline
\end{tabular}

${ }^{\mathrm{T}} \mathrm{Fe}_{2} \mathrm{O}_{3}$ : Total contents of $\mathrm{Fe}_{2} \mathrm{O}_{3}$. 
The total REY concentrations in samples from the Yupanqui Basin ranged from 1057 to $1882 \mathrm{ppm}$ (average $1329 \mathrm{ppm}$ ), gradually increasing with depth. The $\mathrm{CaO}, \mathrm{P}_{2} \mathrm{O}_{5}, \mathrm{Fe}_{2} \mathrm{O}_{3}$, and $\mathrm{MnO}$ contents obtained for the REY-rich muds show positive correlations with depth, whereas the $\mathrm{Al}_{2} \mathrm{O}_{3}, \mathrm{TiO}_{2}$, and $\mathrm{K}_{2} \mathrm{O}$ contents either show no correlation or negative correlations with depth (Figure 4). The $\sum$ REY co-vary positively with $\mathrm{CaO}, \mathrm{P}_{2} \mathrm{O}_{5}, \mathrm{Fe}_{2} \mathrm{O}_{3}$, and $\mathrm{MnO}$, and negatively with $\mathrm{Al}_{2} \mathrm{O}_{3}, \mathrm{TiO}_{2}, \mathrm{~K}_{2} \mathrm{O}, 8 \mathrm{Ce}$ $\left(\mathrm{Ce}_{\mathrm{N}} /\left(\mathrm{La}_{\mathrm{N}} \times \mathrm{Pr}_{\mathrm{N}}\right)^{0.5}\right)$, and $\delta \mathrm{Eu}\left(\mathrm{Eu}_{\mathrm{N}} /\left(\mathrm{Sm}_{\mathrm{N}} \times \mathrm{Gd}_{\mathrm{N}}\right)^{0.5}\right)($ Figure 5$)$. There is no change in the REY patterns of the sediments with the increasing depth. The REY-rich muds have HREE-enriched patterns on Post-Archean Australian Shale (PAAS)-normalized REE diagrams. All of the samples show depletions in $\mathrm{Ce}$, and positive anomalies in Eu and $\mathrm{Y}$ (Figure 6a). In contrast, on seawater-normalized REE diagrams the samples show light rare earth elements (LREE) enriched patterns and positive anomalies in $\mathrm{Ce}$ and $\mathrm{Eu}$ (Figure 7a). 


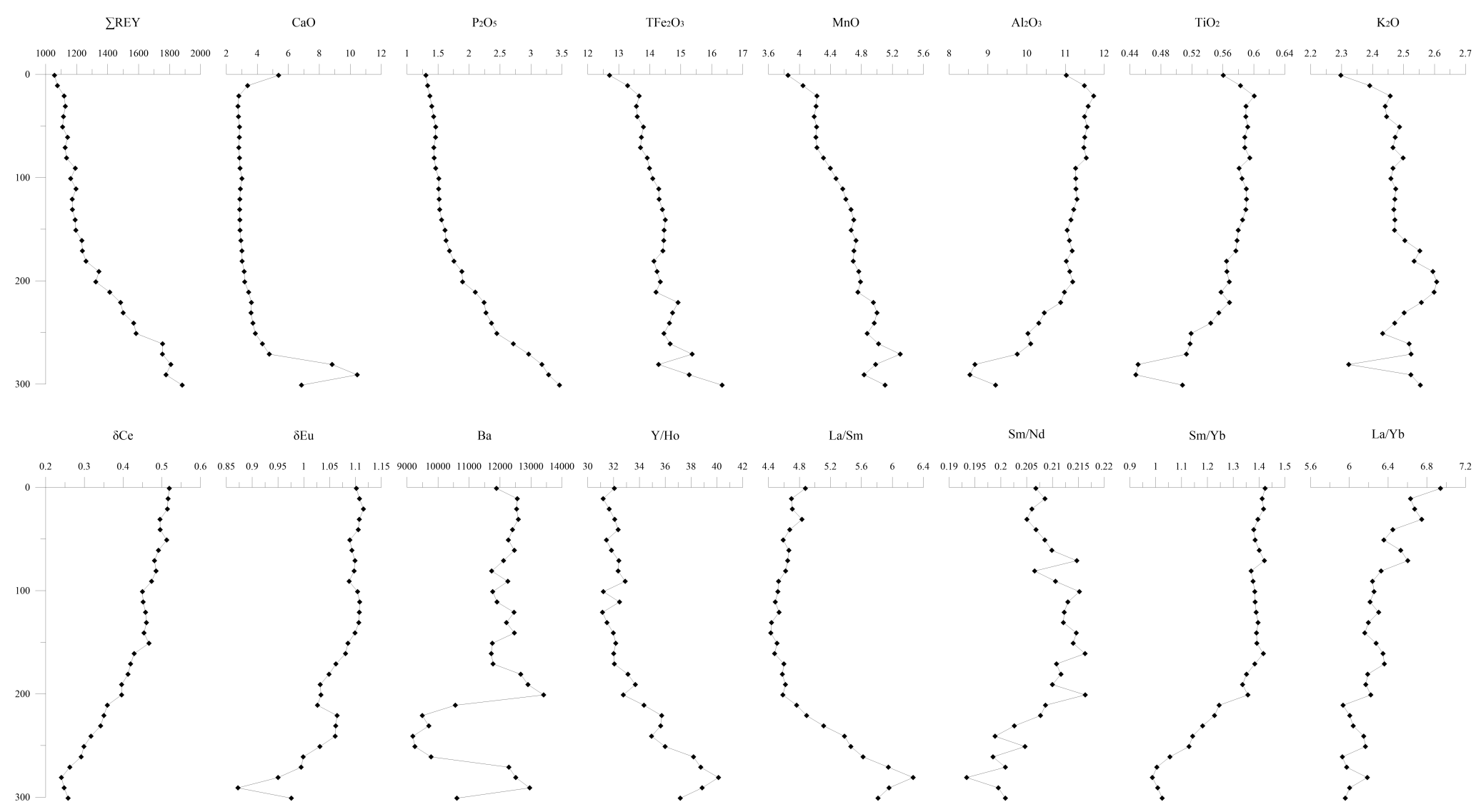

Figure 5. Profiles of REY content and other characteristic parameters of the bulk sediment samples from core S021GC17. 

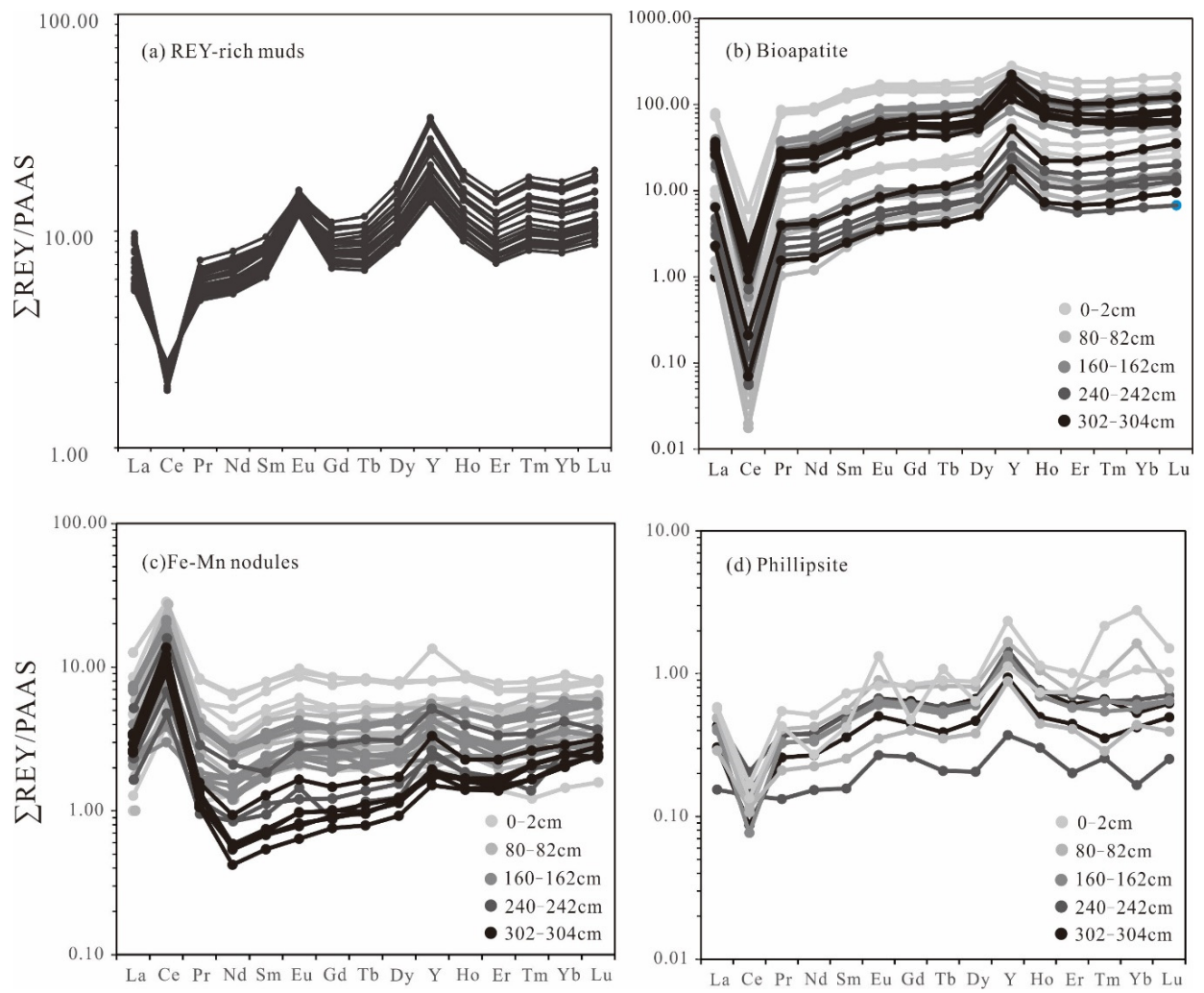

Figure 6. Post-Archean Australian Shale (PAAS)-normalized REY patterns for (a) REY-rich muds, (b) bioapatite, (c) Fe-Mn micronodules, and (d) phillipsite. Normalizing values are from [31].
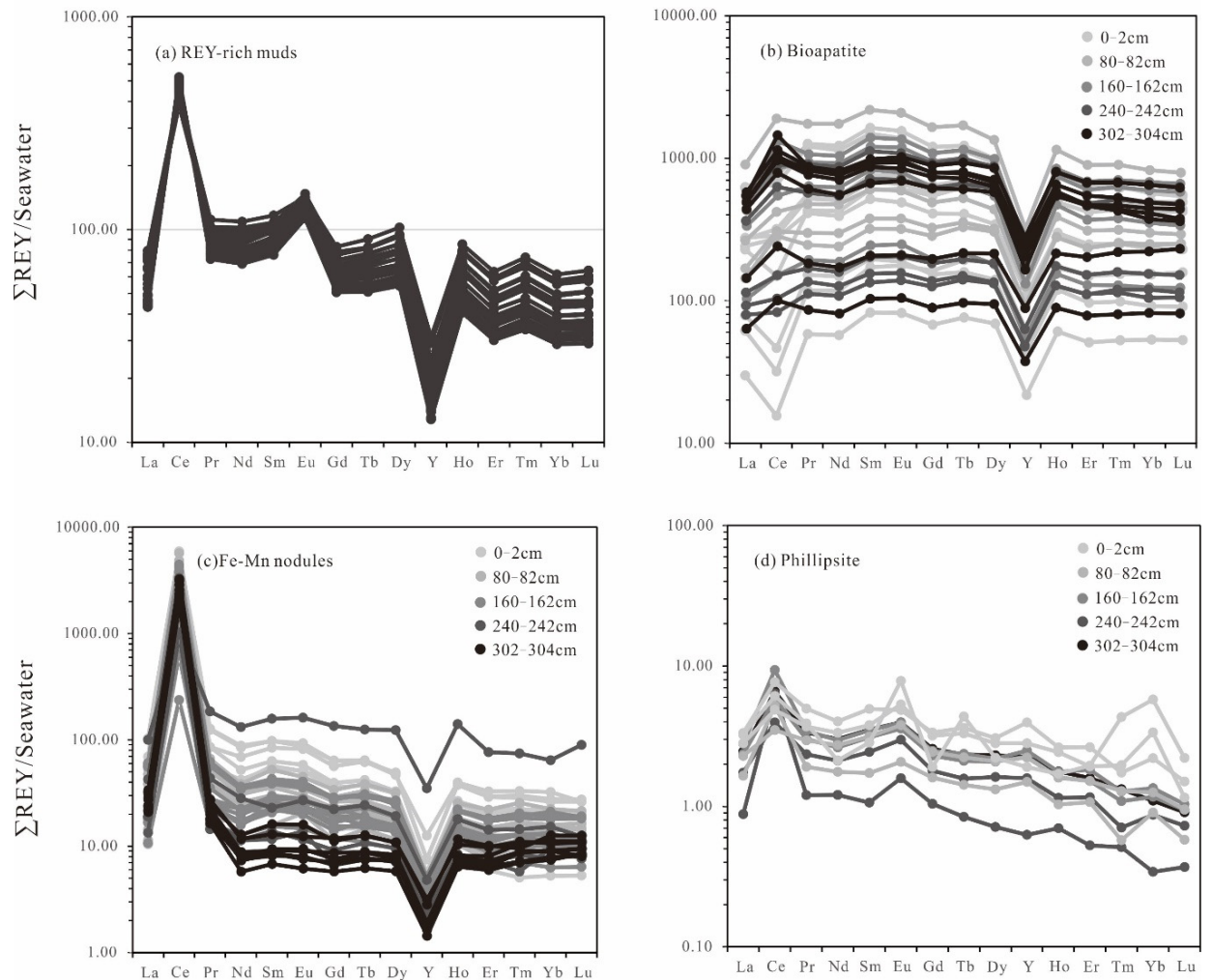

Figure 7. Seawater-normalized REY patterns for (a) REY-rich muds, (b) bioapatite, (c) Fe-Mn micronodules, and (d) phillipsite. Normalizing values are from [32]. 


\subsection{Bulk Sediment Sr-Nd Isotope Compositions}

Considering the compositional regularity of sediments, we selected five samples for Sr-Nd isotope analyses at equal intervals. The results of Sr-Nd isotope analyses of the REY-rich muds (at depths of 0-2, 80-82, 160-162, 240-242, and 302-304 cm) are presented in Table 2.

Table 2. Sr-Nd isotope values for the REY-rich deep-sea sediments and bioapatites in the Yupanqui Basin

\begin{tabular}{|c|c|c|c|c|c|c|c|}
\hline Samples & Samples No. & Depths (cm) & ${ }^{87} \mathrm{Sr} /{ }^{86} \mathrm{Sr}$ & Error (2 s) & ${ }^{143} \mathrm{Nd} /{ }^{144} \mathrm{Nd}$ & Error (2 s) & $\varepsilon N d(0)$ \\
\hline \multirow{4}{*}{$\begin{array}{l}\text { Whole } \\
\text { rock }\end{array}$} & S021GC17-1 & $0-2$ & 0.70951 & 0.00000644 & 0.51245 & 0.00000209 & -3.667305 \\
\hline & S021GC17-2 & $80-82$ & 0.70919 & 0.00000526 & 0.51246 & 0.00000237 & -3.530757 \\
\hline & S021GC17-3 & $160-162$ & 0.71039 & 0.00000681 & 0.51247 & 0.00000199 & -3.355194 \\
\hline & S021GC17-4 & $240-242$ & 0.71081 & 0.00000661 & 0.51248 & 0.00000164 & -3.004069 \\
\hline \multirow{18}{*}{ Bioapatite } & S021GC17-5 & $302-304$ & 0.71084 & 0.00000796 & 0.51249 & 0.00000225 & -2.945548 \\
\hline & 18777-1-1 & $0-2$ & 0.71050 & 0.000134288 & 0.51274 & 0.000032 & 1.945343344 \\
\hline & $18777-1-2$ & $0-2$ & 0.70975 & 0.000231394 & 0.51248 & 0.000032 & -3.14039429 \\
\hline & $18777-1-3$ & $0-2$ & 0.71048 & 0.000686548 & 0.51240 & 0.000066 & -4.57182653 \\
\hline & 18777-1-4 & $0-2$ & 0.70989 & 0.000292885 & 0.51240 & 0.000041 & -4.676482 \\
\hline & 18777-2-1 & $80-82$ & 0.71113 & 0.000226153 & 0.51250 & 0.000033 & -2.76070548 \\
\hline & $18777-2-2$ & $80-82$ & 0.71097 & 0.000298258 & 0.51246 & 0.000041 & -3.53428332 \\
\hline & 18777-3-1 & $160-162$ & 0.71089 & 0.000282621 & 0.51252 & 0.000048 & -2.35227029 \\
\hline & $18777-3-3$ & $160-162$ & 0.71056 & 0.000346566 & 0.51252 & 0.000044 & -2.3695646 \\
\hline & $18777-3-4$ & $160-162$ & 0.71091 & 0.000213473 & 0.51246 & 0.000036 & -3.5659117 \\
\hline & 18777-4-1 & $240-242$ & 0.71158 & 0.000316567 & 0.51251 & 0.000064 & -2.43800703 \\
\hline & $18777-4-2$ & $240-242$ & 0.70914 & 0.000312533 & 0.51266 & 0.000063 & 0.458839981 \\
\hline & $18777-5-1$ & $302-304$ & 0.70985 & 0.000162341 & 0.51255 & 0.000046 & -1.62802313 \\
\hline & $18777-5-2$ & $302-304$ & 0.71008 & 0.000293541 & 0.51258 & 0.000065 & -1.15135076 \\
\hline & $18777-5-3$ & $302-304$ & 0.70961 & 0.000106755 & 0.51246 & 0.000036 & -3.47546196 \\
\hline & $18777-5-4$ & $302-304$ & 0.70891 & 0.00020346 & 0.51255 & 0.000059 & -1.76812022 \\
\hline & $18777-5-5$ & $302-304$ & 0.71100 & 0.000557245 & 0.51268 & 0.000076 & 0.770968358 \\
\hline & $18777-5-6$ & $302-304$ & 0.70941 & 0.000176067 & 0.51250 & 0.000050 & -2.68444508 \\
\hline
\end{tabular}

The initial ${ }^{87} \mathrm{Sr} /{ }^{86} \mathrm{Sr}$ ratios for the REY-rich muds at different depths vary from 0.70919 to 0.71084 , and their initial ${ }^{143} \mathrm{Nd} /{ }^{144} \mathrm{Nd}$ values range from 0.512450 to 0.512487 . The calculated $\varepsilon \mathrm{Nd}(0)$ values range from -3.667305 to -2.945548 . The $\varepsilon \mathrm{Nd}(0)$ values of sediments increase gradually with depth, which is consistent with the trend displayed by $\sum$ REY abundances.

\subsection{EMPA and LA-ICP-MS Major and Trace Element Analyses}

Major element, trace element, and REY compositions of the bioapatite fossils, Fe-Mn micronodules, and phillipsite extracted from the REY-rich muds at depths of 0-2, 80-82, 160-162, 240-242, and 302-304 cm in the core, were measured using EMPA and LA-ICP-MS, and the data are listed in Supplementary Tables S1-S3.

The bioapatite fossils are composed mainly of Ca (29.82-37.27 wt.\%), P (14.55-18.57 wt.\%), and O (28.09-45.04 wt.\%). The total REY contents measured in bioapatites, measured with in situ LA-ICP-MS spot analyses, range from 998 to 22,497 ppm (average 9123 ppm), and do not show any correlation with depths. The REE patterns of bioapatites from different depths are quite uniform. All the samples show clear depletions in $\mathrm{Ce}$, and positive anomalies of $\mathrm{Y}$ in PAAS-normalized REE patterns, in addition to variation in Ce anomalies and negative $\mathrm{Y}$ anomalies in seawater-normalized patterns (Figures $6 \mathrm{~b}$ and $7 \mathrm{~b}$ ).

The Fe-Mn micronodules in the REY-rich sediments from the Yupanqui Basin can be divided into two types according to their composition: type I Fe-Mn micronodules (Figure 3d) and type II Mn micronodules (Figure 3e). The main constituents of the Fe-Mn micronodules are Fe (5.90-14.57 wt.\%), Mn (11.32-31.73 wt.\%), and O (10.48-22.98 wt.\%). The total REY contents of the Fe-Mn micronodules, measured by in situ LA-ICP-MS spot analyses, range from 263 to 3153 ppm (average $1586 \mathrm{ppm}$ ). The Mn micronodules are composed mainly of Mn (22.64-42.24 wt.\%) and O (17.88-35.96 wt.\%), and the contents of Fe are low (0.18-3.93 wt.\%). The REY content in Mn micronodules ranges from 258 to 826 ppm (average 567 ppm). The Fe-Mn micronodules have the similar REY patterns to those of 
the Mn micronodules, with clear Ce positive anomalies in both the PAAS- and seawater-normalized REE patterns (Figures $6 \mathrm{c}$ and $7 \mathrm{c}$ ).

The main constituents of phillipsite are $\mathrm{Si}(21.75-30.15 \mathrm{wt} . \%), \mathrm{Al}(7.34-10.95 \mathrm{wt} . \%)$, and $\mathrm{O}$ (38.82-48.04 wt.\%), with minor amounts of K (1.62-3.05 wt.\%) and Na (0.92-1.60 wt.\%). The REY content in phillipsite ranges from 35 to $127 \mathrm{ppm}$ (average $80 \mathrm{ppm}$ ) and the REY patterns are irregular (Figures $6 \mathrm{~d}$ and $7 \mathrm{~d}$ ).

\subsection{Bioapatite LA-MC-ICP-MS Sr-Nd Isotope Compositions}

Results of the Sr-Nd isotope analyses of bioapatite fossils from different depths $(0-2,80-82,160-162$, $240-242$, and $302-304 \mathrm{~cm}$ ) are presented in Table 2 . The ${ }^{87} \mathrm{Sr} /{ }^{86} \mathrm{Sr}$ ratios for the bioapatites vary from 0.708912 to 0.711583 , and their ${ }^{143} \mathrm{Nd} /{ }^{144} \mathrm{Nd}$ values from 0.512398 to 0.512738 . The calculated $\varepsilon \mathrm{Nd}(0)$ values range from -4.676482 to 1.945343 (average -2.173041 ), and show no correlation with depth.

\section{Discussion}

\subsection{Main Carriers of REY}

\subsubsection{Bioapatites}

Previous studies concluded that biogenic Ca phosphates, such as bioapatite, are the main carriers of REY in deep-sea sediments and that biogenic phosphate occurs mainly in the form of fish teeth [33,34]. Bioapatite has a strong adsorption capacity for REY complexes, and is the main carrier of REYs in the REY-rich muds of the Indian Ocean and the western and central North Pacific $[7,8,14,16]$. Kashiwabara et al. [17] reported XAFS evidence that apatite is the main host phase of La in the Pacific REY-rich mud. Kon et al. [14] conducted in situ geochemical analyses of the bioapatite fossils from the Minami-Torishima area in the western Pacific, and reported that bioapatite fossils are the main REY-hosting phase with the $\sum$ REY range from 9300 to 32,000 ppm. Liao et al. [16] reported the average contents of $6182 \mathrm{ppm}$ of REY in bioapatites from muds of the central North Pacific.

The $\mathrm{CaO}$ and $\mathrm{P}_{2} \mathrm{O}_{5}$ contents of REY-rich muds in the Yupanqui Basin show positive correlations with $\sum$ REY (Figure 5), which suggests that a Ca-phosphate phase is the dominant carrier of REY. The average REY content of bioapatites fossils from the Yupanqui Basin is 9123 ppm (with 8921 ppm exclusion of $\mathrm{Ce}$ ), which confirms that bioapatite is the main REY carrier. The bioapatites are typically fish teeth, and their REY concentrations decrease significantly from the tooth roots to the tips, suggesting that the REYs enter through the roots and diffuse to the tips (Figure 8a,c). This occurs because the dense enamel that coats the bioapatite hinders REY incorporation. REY can only diffuse through the breach at the root, leading to the observed REY gradients [16].

We proposed the assumption that the content of $\mathrm{P}$ in the sediments was entirely supplied by bioapatites. Thus, it can be calculated that the percentage of bioapatite in the sediments is $5.1 \%$. Considering that the average REY content of the bioapatites is $~ 9000 \mathrm{ppm}$ and $\sum R E Y$ in the bulk sediment is $\sim 1000 \mathrm{ppm}$, it can be roughly estimated that the $5 \%$ bioapatite accounts for $\sim 45 \%$ of the REY budget in the bulk sediments. Our results show that bioapatite is the main carrier of REY, even in the eastern South Pacific where significant REY accumulation is related to hydrothermal Fe-Mn (oxyhydr) oxides from the EPR. 

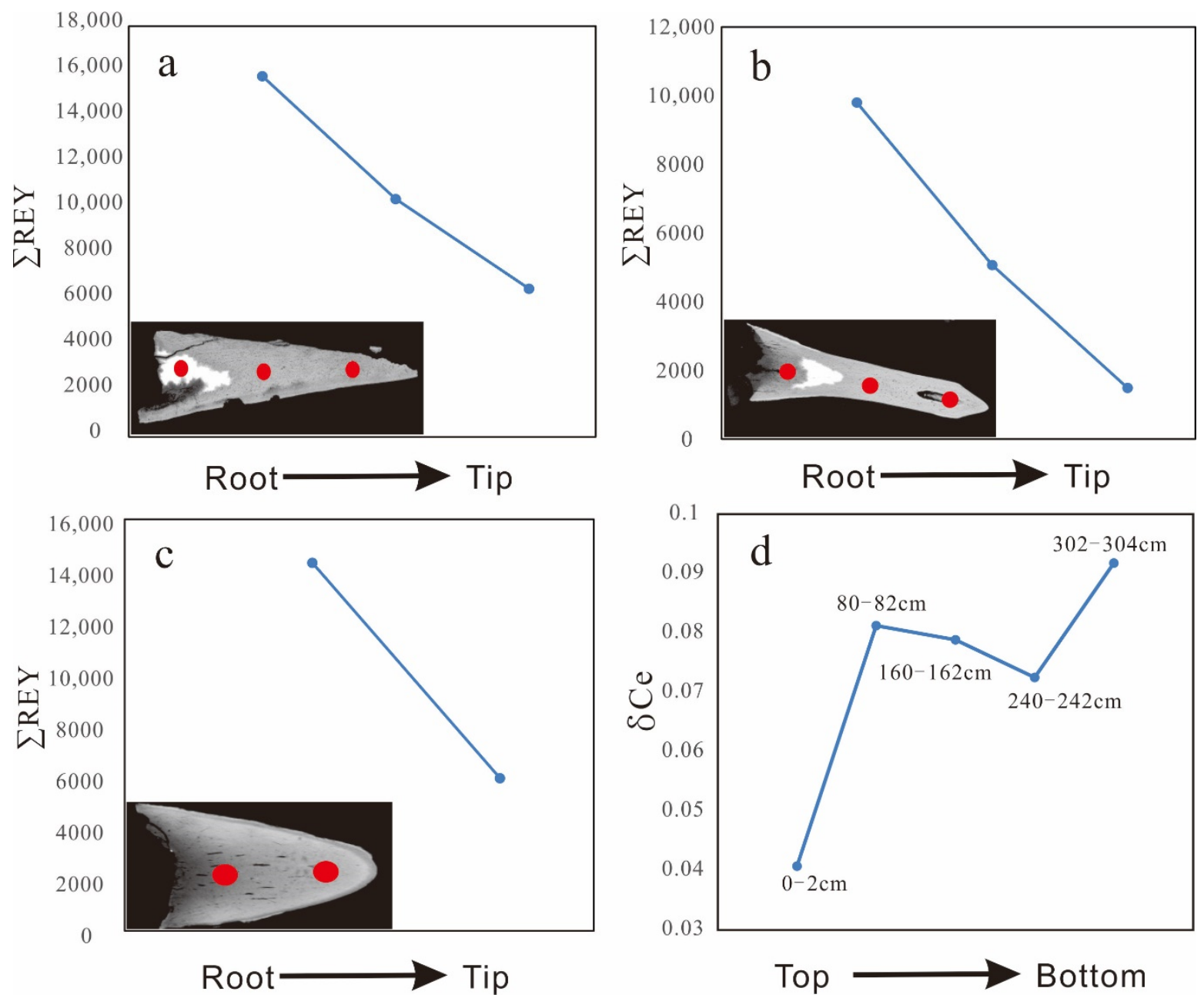

Figure 8. In situ compositional analyses showing the REY and $\delta$ Ce gradients in the bioapatites. (a-c) The contents gradients of REY in the bioapatite fossils. (d) $\delta$ Ce gradients in the bioapatite fossils in different depths.

\subsubsection{Fe-Mn Micronodules}

The $\sum R E Y$ contents of our REY-rich samples not only correlate with $\mathrm{CaO}$ and $\mathrm{P}_{2} \mathrm{O}_{5}$, but also other elements that are not present in bioapatite (e.g., $\mathrm{Fe}_{2} \mathrm{O}_{3}$ and $\mathrm{MnO}$ ) (Figure 5), which suggests that Fe- and Mn-bearing phases also contribute to the formation of the REY-rich muds. The Fe-Mn micronodules in sediments formed as a result of hydrogenetic, diagenetic, and hydrothermal plume processes, and can also act as REY carriers into the sediments $[1,11,13,35,36]$. Fe-Mn micronodules have a low degree of crystallinity and are active scavengers of REYs [1,37-39]. Menendez et al. [40] reported an average REY content of hydrogenetic Fe-Mn micronodules in deep-sea sediments of the Atlantic Ocean of 3620 ppm. Fe-Mn micronodules in REY-rich deep-sea muds recovered from the central North Pacific Ocean contain REY concentrations ranging from 439 to 1654 ppm [16].

To clarify the genetic type of micronodules, their elemental contents of $\mathrm{Mn}, \mathrm{Fe}, \mathrm{Co}, \mathrm{Ni}$, and $\mathrm{Cu}$ were plotted on a ternary discrimination diagram (Figure 9a) [41]. Most of the Fe-Mn micronodules fall into the hydrogenetic and mixed-type fields, whereas the Mn micronodules plot in the diagenetic field, which indicates the co-existence of different genetic mechanisms. Most of the micronodules plot in the transition area between the hydrogenetic and diagenetic fields, and the diagenetic-type (Fe)-Mn micronodules cluster mainly in the oxic diagenesis area on the $100 \times(\mathrm{Zr}+\mathrm{Y}+\mathrm{Ce})$ vs. $15 \times(\mathrm{Cu}+\mathrm{Ni})$ vs. $(\mathrm{Fe}+\mathrm{Mn}) / 4$ diagram proposed by Josso et al. [42] (Figure $9 \mathrm{~b}$ ). The average REY content of $\mathrm{Fe}-\mathrm{Mn}$ micronodules is $1586 \mathrm{ppm}$ (with $490 \mathrm{ppm}$ exclusion of Ce), and the average REY content of 
Mn micronodules is $567 \mathrm{ppm}$ (with $222 \mathrm{ppm}$ exclusion of Ce), which indicates that hydrogenetic micronodules can release REY during the early stages of diagenesis. Given the high proportion of Fe-Mn micronodules in the sediments (15\%), they contribute as much as $22.5 \%$ of the REY content of the sediments. Considering Ce is the least valuable element in REY, the economical values of micronodules in the sediments represent a significant discount.

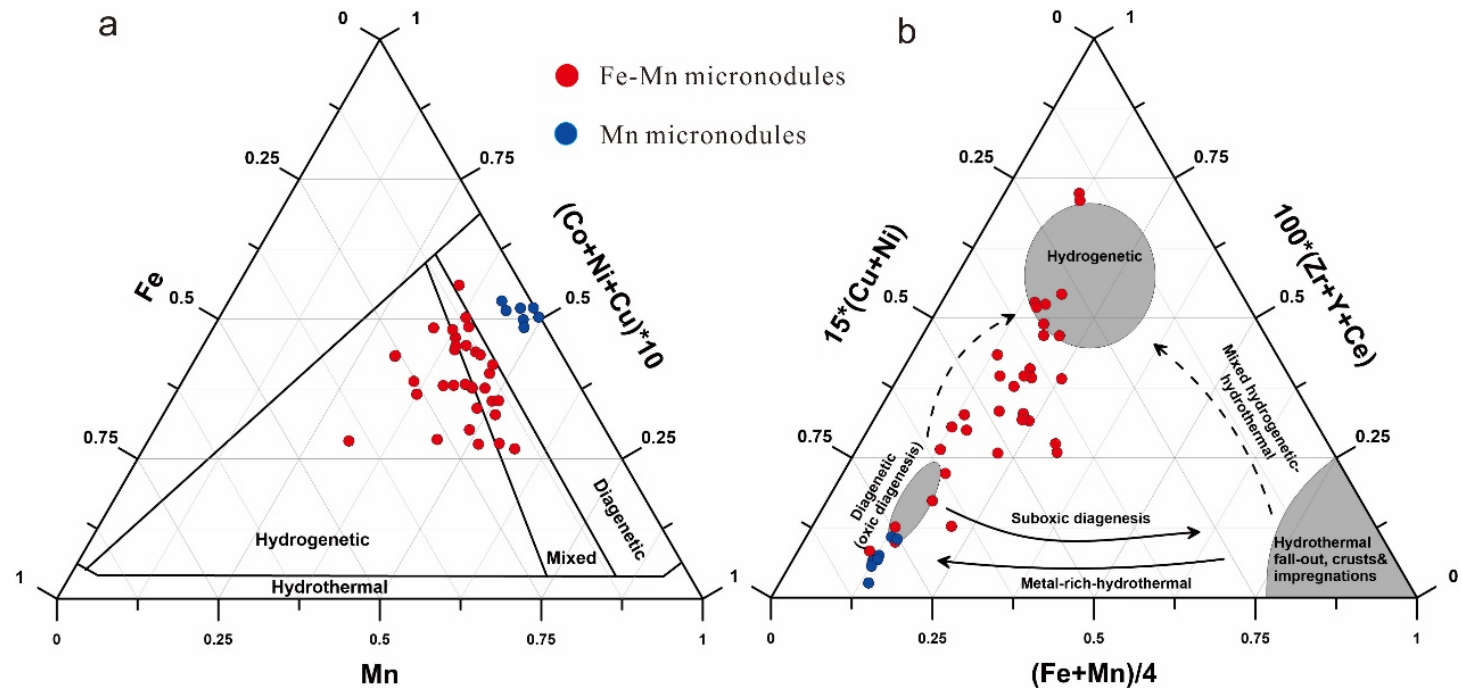

Figure 9. Discrimination diagrams for genetic classification of the Fe-Mn and Mn micronodules in core S021GC17. (a) Ternary Mn vs $(\mathrm{Co}+\mathrm{Ni}+\mathrm{Cu}) \times 10$ vs. Fe discrimination diagram, modified after [37].

(b) $100 \times(\mathrm{Zr}+\mathrm{Y}+\mathrm{Ce})$ vs. $15 \times(\mathrm{Cu}+\mathrm{Ni})$ vs. $(\mathrm{Fe}+\mathrm{Mn}) / 4$ diagram, proposed by [38].

Diagenesis can lead to the remobilization and inter-elemental fractionation of REYs, and these effects often occur in conjunction with redox reactions in the pore waters of sediments [32]. Previous researchers have suggested that REEs were associated primarily with Fe-Mn oxides in the sediments, then subsequently released to pore water during early diagenesis, and ultimately incorporated by bioapatite [43-45]. Variations in the Ce anomalies can be induced by the transfer of REEs from Mn-Fe (oxyhydr)oxides to bioapatite during early diagenesis [45]. Hence, it can be inferred that the REE patterns with positive Ce anomalies in the Fe-Mn micronodules could overprint the primary composition of the bioapatite. The Ce anomalies in our samples of bioapatite show a general increase with depth of burial (Figure 8d), which implies that the uptake of REEs was due to their release from the Fe-Mn micronodules. During early diagenesis, hydrogenetic Fe-Mn micronodules released REYs and associated metals into the pore water, forming the Mn micronodules (Figure 4f,g). Although the complete process remains an open question, the role of pore water in the formation of the REY-rich muds of the eastern South Pacific cannot be neglected.

\subsubsection{Phillipsite}

In a pelagic environment, phillipsite that forms by the alteration of volcanic ash is the one of the most important constituents in the REY-rich muds. Bernat [33] reported that REE contents in fish teeth fossils are two to three orders of magnitude higher than those in phillipsite. Dubinin [46] measured the REE concentrations in phillipsite samples from the Southern Basin of the Pacific, and suggested that phillipsite does not accumulate REY. Kon et al. [14] reported REY contents of 60-170 ppm in phillipsites by in situ mineral analyses.

The REY contents of phillipsites in our samples, measured by in situ LA-ICP-MS spot analyses, range from 35 to $127 \mathrm{ppm}$ (average $80 \mathrm{ppm}$ ), showing that phillipsite is not the main carrier. The requisite for the formation of phillipsite in a marine sediment is an environment with a sufficiently low sedimentation rate, which is similar to the formation of pelagic clays with high REY contents [47]. 
The growth of large phillipsite grains may reflect a low sedimentation rate and long-term exposure of crystals on the seafloor [48]. Hence, phillipsite in the REY-rich muds of the eastern South Pacific is an indicator of low sedimentation rates.

\subsection{The Influence of Hydrothermal Fluids}

Previous researchers suggested that seawater was the main source of the REY in REY-rich sediments, based on the similar REE patterns of seawater and REY-rich muds [7,34,49]. The REY patterns in Figure 6a,b for the muds and the bioapatites do indeed show characteristics similar to those of seawater with their obvious depletions in Ce and positive anomalies of $Y$, and this indicates the REYs were mainly derived mainly from seawater. This is confirmed by the Sr-Nd isotopic compositions of the bulk muds, which are consistent with the isotopic characteristics of seawater in the Pacific (Figure 10). However, the high contents of $\mathrm{Fe}, \mathrm{Mn}, \mathrm{Ba}, \mathrm{Co}$, and $\mathrm{Mo}$ in the REY-rich muds from the Yupanqui basin conceivably imply the influence of other fluids (Table 1a).

It is well known that the East Pacific Rise (EPR) is a fast-spreading mid-ocean ridge [50] that is accompanied by intense hydrothermal activity due to high rates of magma production [11,51]. The dominance of Ce (IV) in the eastern South Pacific could reflect the larger contribution of hydrothermal Fe-Mn oxyhydroxides in this area compared with the central North Pacific [52]. The ${ }^{3} \mathrm{He}$ anomalies in seawater (Figure 1b) show that hydrothermal activity can spread far from a mid-ocean ridge, which is consistent with the distribution of REY-rich muds in the Pacific Ocean [1,11-13]. Hydrothermal fluids are enriched in iron $(\mathrm{Fe})$ and manganese $(\mathrm{Mn})$ by more than $10^{6}$ relative to ambient deep ocean concentrations. Recent studies have also confirmed that Fe-Mn derived from hydrothermal fluid vents can be transported over distances of hundreds or thousands of kilometers $[23,53,54]$. The modern marine environment is generally in an oxic state, as seen in the pronounced negative Ce anomaly of seawater and bioapatite. It is reasonable to suggest that hydrothermal fluids containing considerable amounts of Fe-Mn oxyhydroxide particulates were spread eastwards from the EPR. The oxidized state of the deep-sea environment may contribute Fe-Mn metal ions to the formation of Fe-Mn micronodules, which would promote the enrichment of REY in marine sediments $[55,56]$.

Most of the other REY-rich muds reported in the central North Pacific, the western Pacific, and the Indian Ocean appear to be unrelated to hydrothermal fluids [7-9]. However, the influence of hydrothermal fluids on the sediments in the Yupanqui Basin from the eastern South Pacific Ocean is significant.

The formation of the REY-rich muds in the eastern South Pacific Ocean appears to be associated strongly with hydrothermal activity, as suggested by the positive correlations between $\sum$ REY and both $\mathrm{Fe}_{2} \mathrm{O}_{3}$ and $\mathrm{MnO}$ (Figure 5). The REY-rich sediments from the eastern South Pacific Ocean are enriched in $\mathrm{Fe}, \mathrm{Mn}, \mathrm{Ba}, \mathrm{Co}$, and $\mathrm{Mo}$, which are much higher than the REY-rich sediments from the Indian Ocean, and the western and central North Pacific Ocean [7,8,14,57]. The clear enrichment in these elements is a typical characteristic of hydrothermal fluids and associated precipitates occurring at EPR [37]. The bulk sediments show LREE-enriched patterns and clear Eu positive anomalies in the seawater-normalized $\mathrm{REE}$ diagram, and the ratios of $\mathrm{Fe} / \mathrm{Ti}$ and $\mathrm{Al} /(\mathrm{Al}+\mathrm{Fe}+\mathrm{Mn}$ ) range from 26.4-39.8 (greater than 20) and 0.24-0.33 (less than 0.35 ), which all strongly support the involvement of hydrothermal input during the formation of the REY-rich sediments [37,58]. Moreover, the ${ }^{143} \mathrm{Nd} /{ }^{144} \mathrm{Nd}$ values of the bioapatites are higher than the average values for Pacific seawater, which confirms the overprint of hydrothermal fluids (Figure 10). 

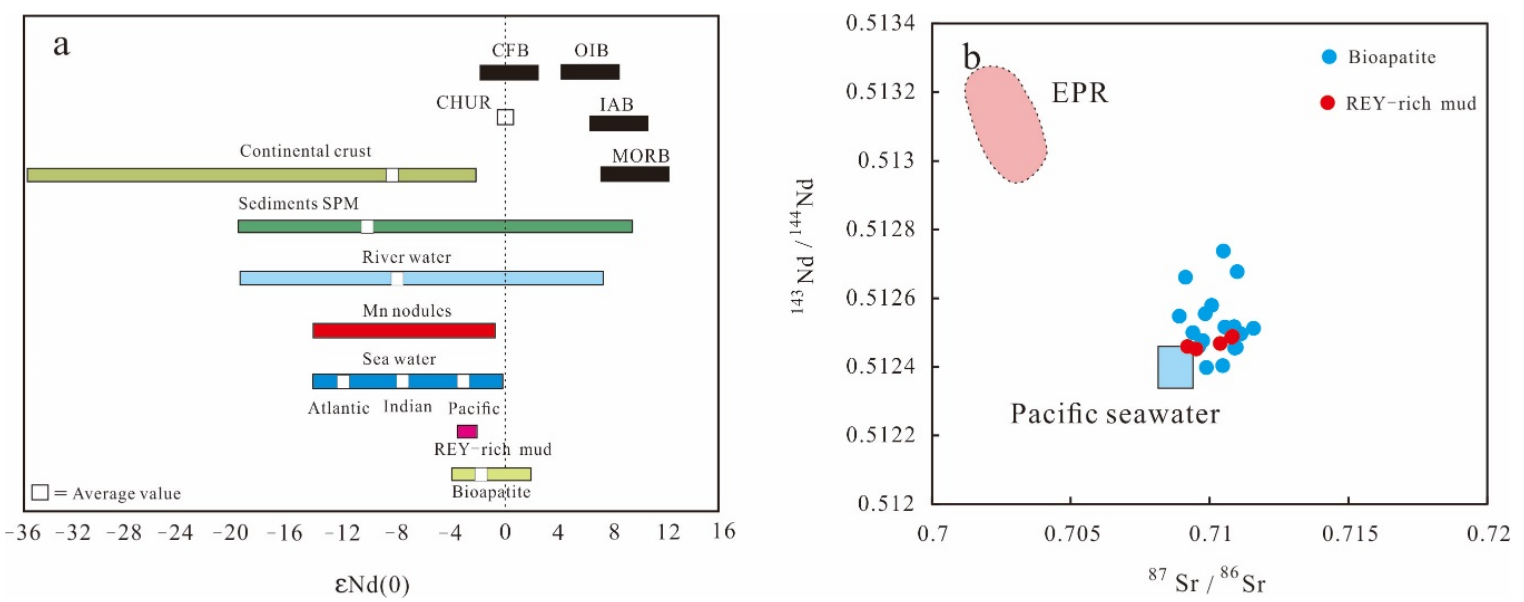

Figure 10. (a) Comparison of $\varepsilon \mathrm{Nd}(0)$ values in the REY-rich muds and bioapatites with various sources. (b) Plot of $\left({ }^{87} \mathrm{Sr} /{ }^{86} \mathrm{Sr}\right)$ vs $\left({ }^{143} \mathrm{Nd} /{ }^{144} \mathrm{Nd}\right)$ for the REY-rich muds and bioapatites. Natural variability of the $\mathrm{Nd}$ isotope composition in different rocks, sediments, and waters, given $\varepsilon \mathrm{Nd}(0)$ values relative to CHUR, modified after [59]. Mid-oceanic ridge basalts proposed by [60]. River water and river sediment $\varepsilon \mathrm{Nd}(0)$ values after [61,62]; seawater $\varepsilon \mathrm{Nd}(0)$ values for the different ocean basins after [63] and [64]. CHUR = chondritic uniform reservoir; $\mathrm{CFB}=$ continental flood basalt; $\mathrm{OIB}=$ ocean island basalt; $\mathrm{IAB}=$ island arc basalt; $\mathrm{MORB}=$ mid-oceanic ridge basalt. EPR/Chile Ridge field proposed by [65].

Thus, we can draw a conclusion that hydrothermal activity plays an important role in the formation of REY-rich muds in the eastern South Pacific. Hydrothermal fluids from the EPR containing considerable amounts of Fe-Mn metal ions have spread thousands of kilometers, precipitating Fe-Mn micronodules in the Yupanqui Basin, where significant quantities of REY are absorbed by the micronodules.

\section{Conclusions}

1. Hydrothermal fluids from the EPR may contribute a large amount of Fe-Mn metal ions, leading to the formation of Fe-Mn micronodules that enable the enrichment of REY in the REY-rich deep-sea sediments.

2. Bioapatite fossils are the main carrier for REYs in the REY-rich deep-sea sediments. REYs enter through the roots of the bioapatites and diffuse to the tips. It can be estimated roughly that the $5 \%$ bioapatites present in the samples account for $\sim 45 \%$ of the REY budget in the analyzed sediments.

3. Ferromanganese micronodules contribute as much as $22.5 \%$ of the REY contents of the sediments, thus also making them an important REY-hosting phase in the eastern South Pacific. During early diagenesis, hydrogenetic Fe-Mn micronodules released REYs into the pore water, which are ultimately absorbed by bioapatites.

4. Phillipsite is not a main host of REY in REY-rich muds from the eastern South Pacific Ocean. This indicates a low sedimentation rate.

Supplementary Materials: The following are available online at http://www.mdpi.com/2075-163X/10/12/1141/s1, Table S1: The major elements content (\%) and trace elements abundance (ppm) of bioapatite from the REY-rich muds in the S021GC17; Table S2: The major elements content (\%) and trace elements abundance (ppm) of micronodules from the REY-rich muds in the S021GC17; Table S3: The major elements content (\%) and trace elements abundance (ppm) of phillipsite from the REY-rich muds in the S021GC17

Author Contributions: Conceptualization, T.Z. and X.S.; investigation, X.S., M.H., X.R., G.Y.; data curation, M.Y., D.B., A.Z.; writing—original draft preparation, T.Z.; writing-review and editing, X.S.; visualization, T.Z.; supervision, X.S.; funding acquisition, X.S. All authors have read and agreed to the published version of the manuscript. 
Funding: This research was supported by the National Key R\&D Program of China (No. 2017YFC0602305), the National "13th Five-Year" Plan Project (DY135-R2-1-01, DY135-R2-1-02), the China Postdoctoral Science Foundation (Grant 2019M660172), Qingdao National Laboratory for Marine Science and Technology (Grant MGQNLM-KF201819), the National Natural Science Foundations of China (No. 41706061, 91858209).

Conflicts of Interest: The authors declare no conflict of interest.

\section{References}

1. Kato, Y.; Fujinaga, K.; Nakamura, K.; Takaya, Y.; Kitamura, K.; Ohta, J.; Toda, R.; Nakashima, T.; Iwamori, H. Deep-sea mud in the Pacific Ocean as a potential resource for rare-earth elements. Nat. Geosci. 2011, 4, 535-539. [CrossRef]

2. Balaram, V.; Banakar, V.K.; Subramanyam, K.S.V.; Roy, P.; Satyanarayanan, M.; Mohan, M.R.; Sawant, S.S. Yttrium and rare earth element contents in seamount cobalt crusts in the Indian Ocean. Curr. Sci. 2012, 103, 1334-1338.

3. Szamałek, K.; Konopka, G.; Zglinicki, K.; Marciniak-Maliszewska, B. New potential source of rare earth elements. Gospod. Surowcami Miner. Miner. Resour. Manag. 2013, 29, 59-76. [CrossRef]

4. Shi, X.F.; Huang, M.; Yu, M. How Much Is Known About Deep-Sea Rare Earths. 10000 Scientific Puzzle; Beijing Science Press: Beijing, China, 2018; pp. 604-605.

5. Wu, C.; Yuan, Z.; Bai, G. Rare earth deposits in China. In Rare Earth Minerals: Chemistry, Origin and Ore Deposits. (The Mineralogical Society Series); Jones, A.P., Wall, F., Williams, C.T., Eds.; Chapman \& Hall: London, UK, 1996; Volume 7, pp. 281-310.

6. Bao, Z.; Zhao, Z. Geochemistry of mineralization with exchangeable REY in the weathering crusts of granitic rocks in South China. Ore Geol. Rev. 2008, 33, 519-535. [CrossRef]

7. Yasukawa, K.; Liu, H.; Fujinaga, K.; Machida, S.; Haraguchi, S.; Ishii, T.; Nakamura, K.; Kato, Y. Geochemistry and mineralogy of REY-rich mud in the eastern Indian Ocean. J. Asian Earth Sci. 2014, 93, 25-36. [CrossRef]

8. Yasukawa, K.; Nakamura, K.; Fujinaga, K.; Machida, S.; Ohta, J.; Takaya, Y.; Kato, Y. Rare-earth, major, and trace element geochemistry of deep-sea sediments in the Indian Ocean: Implications for the potential distribution of REY-rich mud in the Indian Ocean. Geochem. J. 2015, 49, 621-635. [CrossRef]

9. Fujinaga, K.; Yasukawa, K.; Nakamura, K.; Machida, S.; Takaya, Y.; Ohta, J.; Araki, S.; Liu, H.; Usami, R.; Maki, R.; et al. Geochemistry of REY-rich mud in the Japanese exclusive economic zone around Minamitorishima Island. Geochem. J. 2016, 50, 575-590. [CrossRef]

10. Nakamura, K.; Machida, S.; Okino, K.; Masaki, Y.; Iijima, K.M.; Suzuki, K.; Kato, Y. Acoustic characterization of pelagic sediments using sub-bottom profiler data: Implications for the distribution of REY-rich mud in the Minamitorishima EEZ, western Pacific. Geochem. J. 2016, 50, 605-619. [CrossRef]

11. Lupton, J.E. Hydrothermal plumes: Near and far field. In Seafloor Hydrothermal Systems: Physical, Chemical, Biological, and Geological Interactions; Humphris, S.E., Zierenberg, R.A., Mullineaux, L.S., Thomson, R.E., Eds.; American Geophysical Union: Washington, DC, USA, 1995; Volume 91, pp. 317-346.

12. German, C.; Hergt, J.; Palmer, M.; Edmond, J. Geochemistry of a hydrothermal sediment core from the OBS vent-field, $21^{\circ} \mathrm{N}$ East Pacific Rise. Chem. Geol. 1999, 155, 65-75. [CrossRef]

13. Wu, J.; Wells, M.L.; Rember, R. Dissolved iron anomaly in the deep tropical-subtropical Pacific: Evidence for long-range transport of hydrothermal iron. Geochim. Cosmochim. Acta 2011, 75, 460-468. [CrossRef]

14. Kon, Y.; Hoshino, M.; Sanematsu, K.; Morita, S.; Tsunematsu, M.; Okamoto, N.; Yano, N.; Tanaka, M.; Takagi, T. Geochemical characteristics of apatite in heavy REE-rich deep-sea mud from Minami-Torishima area, southeastern Japan. Resour. Geol. 2014, 64, 47-57. [CrossRef]

15. Ohta, J.; Yasukawa, K.; Machida, S.; Fujinaga, K.; Nakamura, K.; Takaya, Y.; Iijima, K.M.; Suzuki, K.; Kato, Y. Geological factors responsible for REY-rich mud in the western North Pacific Ocean: Implications from mineralogy and grain size distributions. Geochem. J. 2016, 50, 591-603. [CrossRef]

16. Liao, J.; Sun, X.; Li, D.; Sa, R.; Lu, Y.; Lin, Z.; Xu, L.; Zhan, R.; Pan, Y.; Xu, H. New insights into nanostructure and geochemistry of bioapatite in REE-rich deep-sea sediments: LA-ICP-MS, TEM, and Z-contrast imaging studies. Chem. Geol. 2019, 512, 58-68. [CrossRef] 
17. Kashiwabara, T.; Toda, R.; Fujinaga, K.; Honma, T.; Takahashi, Y.; Kato, Y. Determination of host phase of lanthanum in deepsea REY-rich mud by XAFS and $\mu$-XRF using high-energy synchrotron radiation. Chem. Lett. 2014, 43, 199-200. [CrossRef]

18. Holler, G.; Marchig, V. Hydrothermal activity on the East Pacific Rise: Stages of development. In Geologisches Jahrbuch; U.S. Department of Energy, Office of Scientific and Technical Information: Oak Ridge, TN, USA, 1990; Volume 75, pp. 3-22.

19. Dick, H.J.; Lin, J.; Schouten, H. An ultraslow-spreading class of ocean ridge. Nature 2003, 426, 405-412. [CrossRef]

20. Faure, V.; Speer, K. Deep circulation in the eastern South Pacific Ocean. J. Mar. Res. 2012, 70, 748-778. [CrossRef]

21. Lupton, J.E.; Craig, H. Excess 3He in oceanic basalts: Evidence for terrestrial primordial helium. Earth Planet. Sci. Lett. 1975, 26, 133-139. [CrossRef]

22. Resing, J.A.; Sedwick, P.N.; German, C.R.; Jenkins, W.J.; Moffett, J.W.; Sohst, B.M.; Tagliabue, A. Basin-scale transport of hydrothermal dissolvedmetals across the South Pacific Ocean. Nature 2015, 523, 200-203. [CrossRef]

23. Fitzsimmons, J.N.; John, S.G.; Marsay, C.M.; Hoffman, C.L.; Nicholas, S.L.; Toner, B.M.; German, C.R.; Sherrell, R.M. Iron persistence in a distal hydrothermal plume supported by dissolved-particulate exchange. Nat. Geosci. 2017, 10, 195-201. [CrossRef]

24. Palma, S.; Silva, N. Epipelagic siphonophore assemblages associated with water masses along a transect between Chile and Easter Island (eastern South Pacific Ocean). J. Plankton Res. 2006, 28, 1143-1151. [CrossRef]

25. Cahill, T.; Isacks, B.L. Seismicity and shape of the subducted Nazca Plate. J. Geophys. Res. Space Phys. 1992, 97, 17503-17529. [CrossRef]

26. Strub, P.T.; Mesias, J.M.; Montecino, V.; Rutllant, J.; Salinas, S. Coastal Ocean Circulation off Western South America. Coast. Segm. (6,E) 1998, 11, 273-313.

27. Emery, W.J.; Meincke, J. Global water masses: Summary and review. Oceanol. Acta 1986, 9, 383-391.

28. Reid, J.L. The shallow salinity minima of the Pacific Ocean. Deep Sea Res. Oceanogr. Abst. 1973, 20, 51-68. [CrossRef]

29. Tanaka, T.; Togashi, S.; Kamioka, H.; Amakawa, H.; Kagami, H.; Hamamoto, T.; Yuhara, M.; Orihasi, Y.; Yoneda, S.; Shimizu, H.; et al. JNdi-1: A neodymium isotopic reference in consistency with LaJolla neodymium. Chem. Geol. 2000, 168, 279-281. [CrossRef]

30. Foster, G.L.; Vance, D. In situ Nd isotopic analysis of geological materials by laser ablation MC-ICP-MS. J. Anal. At. Spectrom. 2006, 21, 288-296. [CrossRef]

31. Taylor, S.R.; McLennan, S.M. The Continental Crust: Its Composition and Evolution; Blackwell Science: Oxford, UK, 1985.

32. Alibo, D.S.; Nozaki, Y. Rare earth elements in seawater: Particle association, shale-normalization, and Ce oxidation. Geochim. Cosmochim. Acta 1999, 63, 363-372. [CrossRef]

33. Bernat, M. Les isotopes de l'uranium et du thorium et les terres rares dans l'environnement marin. Cah. Ostom. Ser. Geol. 1975, 7, 65-83. (In French)

34. Toyoda, K.; Tokonami, M. Diffusion of rare-earth elements in fish teeth from deep-sea sediments. Nature 1990, 345, 607-609. [CrossRef]

35. German, C.R.; Klinkhammer, G.P.; Edmond, J.M.; Mura, A.; Elderfield, H. Hydrothermal scavenging of rare-earth elements in the ocean. Nature 1990, 345, 516-518. [CrossRef]

36. Ren, J.B.; Yao, H.Q.; Zhu, K.C.; He, G.W.; Deng, X.G.; Wang, H.F.; Liu, J.Y.; Fu, P.E.; Yang, S.X. Enrichment mechanism of rare earth elements and yttrium in deep-sea mud of Clarion-Clipperton region. Earth Sci. Front. 2015, 22, 200-211.

37. Bau, M.; Dulski, P. Comparing yttrium and rare earths in hydrothermal fluids from the Mid-Atlantic Ridge: Implications for $\mathrm{Y}$ and REE behavior during near-vent mixing and for the $\mathrm{Y} / \mathrm{Ho}$ ratio of Proteozoic seawater. Chem. Geol. 1999, 155, 77-90.

38. Ohta, A.; Ishii, S.; Sakakibara, M.; Mizuno, A.; Kawabe, I. Systematic correlation of the Ce anomaly with the $\mathrm{Co} /(\mathrm{Ni}+\mathrm{Cu})$ ratio and $\mathrm{Y}$ fractionation from Ho in distinct types of Pacific deep-sea nodules. Geochem. J. 1999, 33, 399-417. [CrossRef]

39. Kanazawa, T.; Sager, W.W.; Escutia, D. Carbonates and bulk sediment geochemistry of ODP Hole 191-1179C. Pangaea 2005. [CrossRef] 
40. Shen, H. Rare earth elements in deep-sea sediments. Geochimica 1990, 19, 340-348, (in Chinese with English abstract).

41. Bonatti, E.; Kraemer, T.; Rydell, H. Classification and genesis of submarine iron-manganese deposits. Papers from a conference on Ferromanganese Deposits on the Ocean Floor. Natl. Sci. Found. 1972, 149-166.

42. Josso, P.; Pelleter, E.; Pourret, O.; Fouquet, Y.; Etoubleau, J.; Cheron, S.; Bollinger, C. A new discrimination scheme for oceanic ferromanganese deposits using high field strength and rare earth elements. Ore Geol. Rev. 2017, 87, 3-15. [CrossRef]

43. Slomp, C.P.; Epping, E.H.G.; Helder, W.; Van Raaphorst, W. A key role for iron-bound phosphorus in authigenic apatite formation in North Atlantic continental platform sediments. J. Mar. Res. 1996, 54, 1179-1205. [CrossRef]

44. Tsandev, I.; Reed, D.C.; Slomp, C.P. Phosphorus diagenesis in deep-sea sediments: Sensitivity to water column conditions and global scale implications. Chem. Geol. 2012, 330, 127-139. [CrossRef]

45. Takahashi, Y.; Hayasaka, Y.; Morita, K.; Kashiwabara, T.; Nakada, R.; Marcus, M.A.; Kato, K.; Tanaka, K.; Shimizu, H. Transfer of rare earth elements (REE) from manganese oxides to phosphates during early diagenesis in pelagic sediments inferred from REE patterns, X-ray absorption spectroscopy, and chemical leaching method. Geochem. J. 2015, 49, 653-674. [CrossRef]

46. Dubinin, A.V. Geochemistry of rare earth elements in oceanic phillipsites. Lithol. Miner. Resour. 2000, 35, 101-108. [CrossRef]

47. Piper, D.Z. Rare earth elements in ferromanganese nodules and other marine phases. Geochim. Cosmochim. Acta 1974, 38, 1007-1022. [CrossRef]

48. Bernat, M.; Church, T.M. Deep-sea phillipsites: Trace geochemistry and modes of formation. ACM Lisp Bulletin 1978, 259-267.

49. Zhang, X.Y.; Deng, H.; Zhang, F.Y.; Zhang, W.Y.; Du, Y.; Jiang, B.B. Enrichment and geochemical characteristics of rare earth elements in deep-sea mud from seamount area of Western Pacific. J. Chin. Soc. Rare Earths 2013, 31, 729-737, (in Chinese with English abstract).

50. Müller, R.D.; Sdrolias, M.; Gaina, C.; Roest, W.R. Age, spreading rates, and spreading asymmetry of the world's ocean crust. Geochem. Geophys. Geosystems 2008, 9. [CrossRef]

51. German, C.R.; Lin, J.; Parson, L.M. Mid-ocean ridges: Hydrothermal interactions between the lithosphere and oceans. Geophys. Monogr. Ser. 2004, 148, 318.

52. Kashiwabara, T.; Toda, R.; Nakamura, K.; Yasukawa, K.; Fujinaga, K.; Kubo, S.; Nozaki, T.; Takahashi, Y.; Suzuki, K.; Kato, Y. Synchrotron X-ray spectroscopic perspective on the formation mechanism of REY-rich muds in the Pacific Ocean. Geochim. Cosmochim. Acta 2018, 240, 274-292. [CrossRef]

53. Bennett, S.A.; Achterberg, E.P.; Connelly, D.P.; Statham, P.J.; Fones, G.R.; German, C.R. The distribution and stabilization of dissolved Fe in deep-sea hydrothermal plumes. Earth Planet. Sci. Lett. 2008, 270, 157-167. [CrossRef]

54. Hawkes, J.A.; Connelly, D.P.; Gledhill, M.; Achterberg, E.P. The stabilisation and transportation of dissolved iron from high temperature hydrothermal vent systems. Earth Planet. Sci. Lett. 2013, 375, 280-290. [CrossRef]

55. Ruhlin, D.E.; Owen, R.M. The rare earth element geochemistry of hydrothermal sediments from the East Pacific Rise: Examination of a seawater scavenging mechanism. Geochim. Cosmochim. Acta 1986, 50, 393-400. [CrossRef]

56. Olivarez, A.M.; Owen, R.M. REE/Fe variations in hydrothermal sediments: Implications for the REE content of seawater. Geochim. Cosmochim. Acta 1989, 53, 757-762. [CrossRef]

57. Sa, R.; Sun, X.; He, G.; Xu, L.; Pan, Q.; Liao, J.; Zhu, K.; Deng, X. Enrichment of rare earth elements in siliceous sediments under slow deposition: A case study of the central North Pacific. Ore Geol. Rev. 2018, 94, 12-23. [CrossRef]

58. Boström, K. Genesis of ferromanganese deposits. In Hydrothermal Processes at Seafloor Spreading Centers; Rona, P.A., Boström, K., Laubier, L., Smith, K.L., Eds.; NATO Conference Series (IV Marine Sciences); Springer: Boston, MA, USA, 1983; Volume 12, pp. 473-489.

59. Piepgras, D.J.; Wasserburg, G.J. Isotopic composition of neodymiuminwaters of the Drake Passage. Science 1982, 217, 207-214. [CrossRef] [PubMed]

60. Goldstein, S.; Hemming, S.R. Long lived isotopic tracers in oceanography. Paleoceanography and ice-sheet dynamics. In Treatise on Geochemistry; Elderfield, H., Turekian, K.K., Eds.; Elsevier: Amsterdam, The Netherlands, 2003; Volume 6, pp. 453-489. 
61. Goldstein, S.J.; Jacobsen, S.B. The Nd and Sr isotopic systematics of river-water dissolved material: Implications for the sources of $\mathrm{Nd}$ and $\mathrm{Sr}$ in seawater. Chem. Geol. Isot. Geosci. Sect. 1987, 66, 245-272. [CrossRef]

62. Goldstein, S.J.; Jacobsen, S.B. Nd and Sr isotopic systematics of river water suspended material: Implications for crustal evolution. Earth Planet. Sci. Lett. 1988, 87, 249-265. [CrossRef]

63. Piepgras, D.J.; Wasserburg, G.J.; Dasch, E.J. The isotopic composition of $\mathrm{Nd}$ in different ocean water masses. Earth Planet. Sci. Lett. 1979, 45, 223-226. [CrossRef]

64. Piepgras, D.J.; Wasserburg, G. Neodymium isotopic variations in seawater. Earth Planet. Sci. Lett. 1980, 50, 128-138. [CrossRef]

65. Bach, W.; Erzinger, J.; Dosso, L.; Bollinger, C.; Bougault, H.; Etoubleau, J.; Sauerwein, J. Unusually large $\mathrm{Nb}-\mathrm{Ta}$ depletions in North Chile ridge basalts at $36^{\circ} 50^{\prime}$ to $38^{\circ} 56^{\prime} \mathrm{S}$ : Major element, trace element, and isotopic data. Earth Planet. Sci. Lett. 1996, 142, 223-240. [CrossRef]

Publisher's Note: MDPI stays neutral with regard to jurisdictional claims in published maps and institutional affiliations.

(C) 2020 by the authors. Licensee MDPI, Basel, Switzerland. This article is an open access article distributed under the terms and conditions of the Creative Commons Attribution (CC BY) license (http://creativecommons.org/licenses/by/4.0/). 